\title{
Probing stratospheric transport and chemistry with new balloon and aircraft observations of the meridional and vertical $\mathrm{N}_{2} \mathrm{O}$ isotope distribution
}

\author{
J. Kaiser ${ }^{1,{ }^{*}}$, A. Engel $^{2}$, R. Borchers ${ }^{3}$, and T. Röckmann ${ }^{1,{ }^{* *}}$ \\ ${ }^{1}$ Atmospheric Physics Division, Max Planck Institute for Nuclear Physics, Heidelberg, Germany \\ ${ }^{2}$ Institute for Atmosphere and Environment, J. W. Goethe University, Frankfurt, Germany \\ ${ }^{3}$ Planets and Comets Department, Max Planck Institute for Solar System Research, Katlenburg-Lindau, Germany \\ * now at: School of Environmental Sciences, University of East Anglia, Norwich, UK \\ ** now at: Institute for Marine and Atmospheric Research Utrecht, Utrecht University, The Netherlands
}

Received: 16 January 2006 - Published in Atmos. Chem. Phys. Discuss.: 29 May 2006

Revised: 14 August 2006 - Accepted: 17 August 2006 - Published: 30 August 2006

\begin{abstract}
A comprehensive set of stratospheric balloon and aircraft samples was analyzed for the position-dependent isotopic composition of nitrous oxide $\left(\mathrm{N}_{2} \mathrm{O}\right)$. Results for a total of 220 samples from between 1987 and 2003 are presented, nearly tripling the number of mass-spectrometric $\mathrm{N}_{2} \mathrm{O}$ isotope measurements in the stratosphere published to date. Cryogenic balloon samples were obtained at polar (Kiruna/Sweden, $68^{\circ} \mathrm{N}$ ), mid-latitude (southern France, $44^{\circ} \mathrm{N}$ ) and tropical sites (Hyderabad/India, $18^{\circ} \mathrm{N}$ ). Aircraft samples were collected with a newly-developed whole air sampler on board of the high-altitude aircraft M55 Geophysica during the EUPLEX 2003 campaign. For mixing ratios above $200 \mathrm{nmol} \mathrm{mol}^{-1}$, relative isotope enrichments $(\delta$ values) and mixing ratios display a compact relationship, which is nearly independent of latitude and season and which can be explained equally well by Rayleigh fractionation or mixing. However, for mixing ratios below $200 \mathrm{nmol} \mathrm{mol}^{-1}$ this compact relationship gives way to meridional, seasonal and interannual variations. A comparison to a previously published mid-latitude balloon profile even shows large zonal variations, justifying the use of three-dimensional (3-D) models for further data interpretation.
\end{abstract}

In general, the magnitude of the apparent fractionation constants (i.e., apparent isotope effects) increases continuously with altitude and decreases from the equator to the North Pole. Only the latter observation can be understood qualitatively by the interplay between the time-scales of $\mathrm{N}_{2} \mathrm{O}$ photochemistry and transport in a Rayleigh fractionation framework. Deviations from Rayleigh fractionation behavior also occur where polar vortex air mixes with nearly $\mathrm{N}_{2} \mathrm{O}$-free upper stratospheric/mesospheric air (e.g., during the boreal winters of 2003 and possibly 1992). Aircraft observations

Correspondence to: J. Kaiser

(J.Kaiser@uea.ac.uk) in the polar vortex at mixing ratios below $200 \mathrm{nmol} \mathrm{mol}^{-1}$ deviate from isotope variations expected for both Rayleigh fractionation and two-end-member mixing, but could be explained by continuous weak mixing between intravortex and extravortex air (Plumb et al., 2000). However, it appears that none of the simple approaches described here can capture all features of the stratospheric $\mathrm{N}_{2} \mathrm{O}$ isotope distribution, again justifying the use of 3-D models. Finally, correlations between ${ }^{18} \mathrm{O} /{ }^{16} \mathrm{O}$ and average ${ }^{15} \mathrm{~N} /{ }^{14} \mathrm{~N}$ isotope ratios or between the position-dependent ${ }^{15} \mathrm{~N} /{ }^{14} \mathrm{~N}$ isotope ratios show that photo-oxidation makes a large contribution to the total $\mathrm{N}_{2} \mathrm{O}$ sink in the lower stratosphere (possibly up to $100 \%$ for $\mathrm{N}_{2} \mathrm{O}$ mixing ratios above $300 \mathrm{nmol} \mathrm{mol}^{-1}$ ). Towards higher altitudes, the temperature dependence of these isotope correlations becomes visible in the stratospheric observations.

\section{Introduction}

Stratospheric $\mathrm{N}_{2} \mathrm{O}$ is enriched in the heavy nitrogen and oxygen isotopes $\left({ }^{15} \mathrm{~N},{ }^{17} \mathrm{O},{ }^{18} \mathrm{O}\right)$ relative to tropospheric $\mathrm{N}_{2} \mathrm{O}$. This enrichment is caused by kinetic isotope fractionation in the stratospheric sink reactions, i.e., ultraviolet photolysis (R1) and reaction with electronically excited oxygen atoms, $\mathrm{O}\left({ }^{1} \mathrm{D}\right)$ (Reactions R2a+b):

$$
\begin{aligned}
& \mathrm{N}_{2} \mathrm{O}+h v \rightarrow \mathrm{N}_{2}+\mathrm{O}\left({ }^{1} \mathrm{D}\right) \quad(185 \mathrm{~nm}<\lambda<225 \mathrm{~nm}) \\
& \mathrm{N}_{2} \mathrm{O}+\mathrm{O}\left({ }^{1} \mathrm{D}\right) \rightarrow \mathrm{NO}+\mathrm{NO} \\
& \rightarrow \mathrm{N}_{2}+\mathrm{O}_{2}
\end{aligned}
$$

Reaction (R2) is also called "photo-oxidation", even though, strictly speaking, $\mathrm{O}\left({ }^{1} \mathrm{D}\right)$ only reacts as an oxidant in the NO+NO channel. Reaction (R1) accounts for about $90 \%$

Published by Copernicus GmbH on behalf of the European Geosciences Union. 
of the total sink, whereas Reactions (R2a) and (R2b) contribute $6 \%$ and $4 \%$, respectively (Minschwaner et al., 1993).

The dependence of the $\mathrm{N}_{2} \mathrm{O}$ absorption spectrum on isotopic composition was already studied in the early 1980s (Selwyn and Johnston, 1981), but the relevance of this isotope effect for the ${ }^{15} \mathrm{~N}$ enrichment observed in a single stratospheric $\mathrm{N}_{2} \mathrm{O}$ sample (Moore, 1974) was not recognized before the early 1990s (Yoshida et al., 1990). Two additional stratospheric air samples analyzed by Kim and Craig (1993) substantiated the $\mathrm{N}_{2} \mathrm{O}$ isotope enrichment for both ${ }^{15} \mathrm{~N}$ and ${ }^{18} \mathrm{O}$, but the results may have been impaired by $\mathrm{CO}_{2}$ contamination (Rahn and Wahlen, 1997). Laboratory studies of isotope fractionation in $\mathrm{N}_{2} \mathrm{O}$ photolysis at $185 \mathrm{~nm}$ and during "photo-oxidation" found only small oxygen isotope effects (Johnston et al., 1995), in apparent contradiction to the stratospheric findings. However, additional high-quality field data from Rahn and Wahlen (1997) were accompanied by theoretical predictions of an increase of the photolytic isotope effect towards longer, stratospherically more relevant wavelengths (Yung and Miller, 1997). Laboratory measurements of isotope effects between 193 and $208 \mathrm{~nm}$ quickly showed that these theoretical predictions were qualitatively correct, but at least a factor of two too small in magnitude (Rahn et al., 1998). Analytical advances in the late 1990s then allowed the position-dependent measurement of the nitrogen isotope distribution between the terminal and central nitrogen atoms in $\mathrm{N}_{2} \mathrm{O}$ (Brenninkmeijer and Röckmann, 1999; Esler et al., 2000; Toyoda and Yoshida, 1999). The new techniques were immediately adopted for extensive laboratory measurements of kinetic isotope effects during photolysis (summarized in Kaiser et al., 2003b and von Hessberg et al., 2004) and the reaction of $\mathrm{N}_{2} \mathrm{O}$ with $\mathrm{O}\left({ }^{1} \mathrm{D}\right)$ (Kaiser et al., 2002a; Toyoda et al., 2004). Our present understanding of these isotope effects can be considered to be very good.

The new analytical techniques were used for further stratospheric measurements, as evidenced by six publications (Griffith et al., 2000; Park et al., 2004; Röckmann et al., 2001; Toyoda et al., 2001, 2004; Yoshida and Toyoda, 2000). The paper by Toyoda et al. (2004) also contains the data from the earlier two papers by the same principal authors. All but the Fourier transform infrared-spectra (FTIR) of Griffith et al. (2000) were analyzed by isotope ratio mass-spectrometry (IRMS) of discrete whole-air samples, either obtained by aircraft (Park et al., 2004) or from balloon platforms. A total of 32 samples were analyzed by Park et al. (2004). Toyoda and co-workers analyzed 72 samples. In the following, we present data from an additional 132 balloon and 88 aircraft samples, obtained at latitudes between $18^{\circ} \mathrm{N}$ and $80^{\circ} \mathrm{N}$. A subset of ten tropical, one mid-latitude, and eight polar samples were already included in our previous paper (Röckmann et al., 2001). Importantly, we show two balloon profiles from Hyderabad/India at $18^{\circ} \mathrm{N}$, the only existing stratospheric $\mathrm{N}_{2} \mathrm{O}$ isotope measurements at low latitudes. The tropics are important because there, the upwelling branches of the Hadley and the Brewer-Dobson circulation lead to net air mass transport from the troposphere to the stratosphere. Thus, the "youngest" stratospheric air masses are found in the tropics, where they undergo rapid photochemical processing. However, even though the geographic location of Hyderabad is in the tropics, this does not necessarily mean that the air mass sampled there can be considered tropical with respect to the atmospheric circulation system, as explained in Sect. 3.1. Further samples from the "deep" tropics are needed to fully characterize the isotopic composition of $\mathrm{N}_{2} \mathrm{O}$ in upwelling tropical air.

We will use tracer-tracer diagrams of stratospheric $\mathrm{N}_{2} \mathrm{O}$ isotope and concentration measurements to investigate transport, mixing and photochemical processes (Sects. 3.2 and 3.3). Using correlations between isotope enrichments and mixing ratios, it will be shown to what extent the influence of chemistry and transport on $\mathrm{N}_{2} \mathrm{O}$ isotope distributions can be interpreted in the framework of a one-dimensional reactionadvection-diffusion regime (Sect. 3.2), or as two-end member mixing relationships (Sect. 3.3). Meridional and seasonal variations in apparent fractionation constants are identified, followed by the discussion of possible mechanisms explaining these variations (Sects. 3.2 to 3.4). We also show that the relationship between different intramolecular isotopic signatures of $\mathrm{N}_{2} \mathrm{O}$ varies with altitude, giving insights into the contribution of individual $\mathrm{N}_{2} \mathrm{O}$ sinks at different stratospheric levels (Sect. 3.5).

\section{Experimental methods}

\subsection{Sample collection}

Upper tropospheric and stratospheric balloon air samples from altitudes between 6 and $34 \mathrm{~km}$ were collected at one tropical (Hyderabad/India, $17.5^{\circ} \mathrm{N}$ ), two mid-latitude (Aire sur l'Adour/France, $43.7^{\circ} \mathrm{N}$; Gap/France, $44.4^{\circ} \mathrm{N}$ ), and one polar site (Kiruna/Sweden, $\left.67.9^{\circ} \mathrm{N}\right)$. We analyzed a total of 132 samples, which were collected using balloon-borne cryogenic whole air samplers during 12 launches between March 1987 and March 2003. The first eight profiles between 1987 and 1999 were acquired with the air sampler of the former Max Planck Institute (MPI) for Aeronomy, Katlenburg-Lindau/Germany (now: MPI for Solar System Research). The final four profiles were performed by the BONBON air sampler jointly developed by the Research Centre Jülich/Germany and the Institute for Meteorology and Geophysics (now: Institute for Atmosphere and Environment) at the J. W. Goethe University, Frankfurt/Germany. Both air samplers consist of 15 electro-polished stainless steel tubes immersed into liquid neon at a temperature of $27 \mathrm{~K}$, but differ especially in the intake design (see Schmidt et al., 1987, for details). The sampling tubes have an internal volume of about $500 \mathrm{~cm}^{3}$ and usually contain between 2.5 and $25 \mathrm{dm}^{3}$ of sample at standard temperature and pressure (STP), corresponding to pressures between 0.54 and $5.4 \mathrm{MPa}$ 
at $20^{\circ} \mathrm{C}$. The altitude-resolution is about $1 \mathrm{~km}$, sampling latitudes are essentially invariant, and longitude variations can be a few degrees, depending on the prevailing zonal winds during sample collection.

Aircraft samples were collected from the high-latitude research aircraft M55 Geophysica during the EUPLEX (European Polar Stratospheric Cloud and Lee Wave Experiment) campaign in January/February 2003 in Kiruna/Sweden $\left(67.9^{\circ} \mathrm{N}\right)$. A total of 88 samples was analyzed for their $\mathrm{N}_{2} \mathrm{O}$ isotopic composition. The samples were collected using a new whole air sampler developed at the MPI for Nuclear Physics, Heidelberg/Germany. The sampler uses trace-gas clean metal bellows pumps to collect up to 20 samples into electro-polished stainless steel flasks of $2 \mathrm{dm}^{3}$ internal volume. At a pressure of about 0.3 to $0.4 \mathrm{MPa}$, this gives sample amounts of 6 to $8 \mathrm{dm}^{3}$ (STP). The samples span a smaller altitude range ( 8 to $20 \mathrm{~km}$ ) than individual balloon profiles, but larger latitude $\left(65.6\right.$ to $\left.80^{\circ} \mathrm{N}\right)$ and longitude $\left(9.1\right.$ to $\left.48.8^{\circ} \mathrm{E}\right)$ bands.

Table 1 summarizes sampling dates and locations of all published isotope measurements of stratospheric $\mathrm{N}_{2} \mathrm{O}$, including the 220 samples analyzed for this paper. $\mathrm{N}_{2} \mathrm{O}$ is usually zonally well-mixed, but for completeness we show both latitude and longitude of the sampling locations, where available.

2.2 Isotope analysis and measurements of $\mathrm{N}_{2} \mathrm{O}$ mixing ratios

The position-dependent isotopic composition of $\mathrm{N}_{2} \mathrm{O}$ was measured as described previously (Röckmann et al., 2003b). We assign a locant 1 to the terminal nitrogen atom in $\mathrm{N}_{2} \mathrm{O}$ and a locant 2 to the central nitrogen atom, so that the corresponding relative isotope enrichments ( $\delta$ values) are designated ${ }^{1} \delta^{15} \mathrm{~N}$ and ${ }^{2} \delta^{15} \mathrm{~N}$. Other authors use a locant $\beta$ for the terminal and $\alpha$ for the central nitrogen atom (Yoshida and Toyoda, 2000). $\delta$ values are defined as relative deviations of the isotope ratio of a sample to a reference isotope ratio:

$\delta=\frac{R_{\text {sample }}}{R_{\text {reference }}}-1$

The isotope ratio $R$ is defined as the abundance ratio of the heavier isotope and the lighter isotope, i.e., ${ }^{15} \mathrm{~N}$ and ${ }^{14} \mathrm{~N}$ in the case of nitrogen and ${ }^{18} \mathrm{O}$ and ${ }^{16} \mathrm{O}$ in the case of oxygen. Variations of the ${ }^{17} \mathrm{O} /{ }^{16} \mathrm{O}$ isotope ratio were not measured, but for data reduction purposes, we assume that stratospheric $\mathrm{N}_{2} \mathrm{O}$ has the same small relative ${ }^{17} \mathrm{O}$ excess of $\Delta{ }^{17} \mathrm{O}=0.9 \%$ 。 as tropospheric $\mathrm{N}_{2} \mathrm{O}$ (Kaiser et al., 2003a). This assumption follows theoretical considerations (Kaiser and Röckmann, 2005), but the possible error introduced by this assumption is small because the ${ }^{17} \mathrm{O}$ correction to the $\delta^{15} \mathrm{~N}$ values is only about $-0.1 \Delta^{17} \mathrm{O}$ for ${ }^{2} \delta^{15} \mathrm{~N}$ and $-0.05 \Delta^{17} \mathrm{O}$ for average $\delta^{15} \mathrm{~N}=\left({ }^{1} \delta^{15} \mathrm{~N}+{ }^{2} \delta^{15} \mathrm{~N}\right) / 2$.

Generally, the reference isotope ratios are those of air- $\mathrm{N}_{2}$ for nitrogen and Vienna Standard Mean Ocean Water (VS-
MOW) for oxygen isotope measurements. However, in the present paper we frequently deviate from this convention and express the stratospheric isotope ratios relative to those of tropospheric $\mathrm{N}_{2} \mathrm{O}$. Thus, the individual isotope signatures are more easily intercomparable. Moreover, assigning a specific position-dependent nitrogen isotope scale is problematic at the present time because two significantly different absolute isotope calibrations exist and the discrepancies have not been resolved yet (Kaiser et al., 2004a; Toyoda and Yoshida, 1999). To compare the isotope profiles for individual years directly, we apply a further normalization by correcting for the temporal trend in the isotope ratio of tropospheric $\mathrm{N}_{2} \mathrm{O}$ and the age of stratospheric air (Sect. 3.2). Furthermore, we can study variations in the ratio between position-dependent isotope signatures for individual samples in order to separate isotope variations due to transport from those due to changing chemistry (Sect. 3.5).

In practice, we base our isotope measurements on a tank of tropospheric $\mathrm{N}_{2} \mathrm{O}$, which was collected on 15 March 2002 at Mount Schauinsland in southwest Germany (SIL- $\mathrm{N}_{2} \mathrm{O}$ ). Its isotope composition was determined by offline $\mathrm{N}_{2} \mathrm{O}$ isotope analysis (Kaiser et al., 2003a) and found to be $\delta^{15} \mathrm{~N}=(6.6 \pm 0.1) \% o,{ }^{1} \delta^{15} \mathrm{~N}=(-16.0 \pm 0.2) \%$, ${ }^{2} \delta^{15} \mathrm{~N}=(29.1 \pm 0.2) \%$ and $\delta^{18} \mathrm{O}=(44.6 \pm 0.1) \%$ o. In order to avoid large nonlinearity corrections (see Röckmann et al., 2003b), we adjusted the extraction time of the trace gas preconcentration system using a previously measured $\mathrm{N}_{2} \mathrm{O}$ mixing ratio value so that the peak area matched that of the Schauinsland reference tank sample (about $4.4 \mathrm{Vs}$ for the $\mathrm{N}_{2} \mathrm{O}^{+}$peak, corresponding to $2.0 \mathrm{nmol}$ of $\mathrm{N}_{2} \mathrm{O}$ ). In cases where the available sample amount was limited, this was not always possible, but even then, the necessary non-linearity corrections were at most $0.5 \%$ for $\delta^{15} \mathrm{~N}$ and $\delta^{18} \mathrm{O}$ and less than $1.5 \%$ for ${ }^{1} \delta^{15} \mathrm{~N}$ and ${ }^{2} \delta^{15} \mathrm{~N}$. This concerned samples from the high latitudes and/or altitudes with correspondingly low mixing ratios and high $\delta$ values, so that the relative error due to this non-linearity correction is small. For analyses of the $\mathrm{N}_{2} \mathrm{O}^{+}$molecular ion, sample sizes ranged from 0.3 to $2.1 \mathrm{nmol}(0.5$ to $4.6 \mathrm{Vs})$ with an average of $(1.7 \pm 0.4) \mathrm{nmol}$ or $(3.6 \pm 0.9)$ Vs. For the $\mathrm{NO}^{+}$fragment ion, which is used for position-dependent ${ }^{15} \mathrm{~N}$ analysis, the range was 1.0 to $7.0 \mathrm{nmol}(0.4$ to $2.8 \mathrm{Vs})$ with an average of $(4.4 \pm 1.0) \mathrm{nmol}$ or $(1.7 \pm 0.4)$ Vs. A larger amount of sample was used for $\mathrm{NO}^{+}$ fragment analysis because the relative abundance of $\mathrm{NO}^{+}$ in the $\mathrm{N}_{2} \mathrm{O}$ mass spectrum is only about $20 \%$ of the $\mathrm{N}_{2} \mathrm{O}^{+}$ molecular ion for the mass spectrometers and ion source configurations we were using.

All isotope analyses were performed on a Finnigan Delta Plus XL isotope ratio mass spectrometer except for the last balloon flight on 6 March 2003, which was analyzed on a Delta Plus XP mass spectrometer. The Delta Plus XP allows simultaneous registering of mass-to-charge ratios 30 , $31,44,45$, and 46 , which has the advantage of requiring only a single extraction/injection of sample for analysis. The reproducibility for a $2 \mathrm{nmol} \mathrm{N}_{2} \mathrm{O}$ sample was about $0.15 \%$ or 
Table 1. Sampling dates and locations of past upper tropospheric and stratospheric $\mathrm{N}_{2} \mathrm{O}$ isotope measurements. Unknown dates are marked by x's. Most isotope measurements have been performed by isotope-ratio mass spectrometry (IRMS), except for the Fourier transform infrared spectroscopic (FTIR) data of Griffith et al. (2000).

\begin{tabular}{|c|c|c|c|c|c|c|}
\hline $\begin{array}{l}\text { Location } \\
\text { or campaign }\end{array}$ & Date & $\begin{array}{l}\text { Latitude } \\
{ }^{\circ} \mathrm{N}\end{array}$ & $\begin{array}{l}\text { Longitude } \\
{ }^{\circ} \mathrm{E}\end{array}$ & $\begin{array}{l}\text { Altitude } \\
\mathrm{km}\end{array}$ & Samples & Reference \\
\hline Mildura, Australia & xx xxx 197x & -34 & 142 & 21 & 1 & Moore (1974) \\
\hline \multirow[t]{2}{*}{ Kiruna, Sweden } & 10 Jan 1988 & 68 & 20 & 13 & 1 & \multirow[t]{2}{*}{ Kim and Craig (1993) } \\
\hline & 10 Feb 1988 & 68 & 20 & 18 & 1 & \\
\hline Kiruna, Sweden & 10 Feb 1988 & 68 & 20 & $14+23$ & 2 & \multirow[t]{4}{*}{ Rahn and Wahlen (1997) } \\
\hline \multirow[t]{3}{*}{ Contiguous USA } & 27 Jan 1988 & 46 & -94 & 17 & 1 & \\
\hline & 10 May 88 & $39-45$ & -94 & $17+18$ & 2 & \\
\hline & 12 April 1989 & $40-48$ & -94 & $15+17$ & 2 & \\
\hline \multirow[t]{6}{*}{ Fort Sumner, USA } & 14 Sep 1992 & 34 & -104 & $18-35$ & 1 profile & \multirow[t]{9}{*}{ Griffith et al. (2000) } \\
\hline & 15 Sep 1992 & 34 & -104 & $18-35$ & 1 profile & \\
\hline & 25 Sep 1993 & 34 & -104 & $18-35$ & 1 profile & \\
\hline & 26 Sep 1993 & 34 & -104 & $18-35$ & 1 profile & \\
\hline & 22 May 1994 & 34 & -104 & $18-35$ & 1 profile & \\
\hline & 28 Sep 96 & 34 & -104 & $18-35$ & 1 profile & \\
\hline \multirow[t]{2}{*}{ Fairbanks, USA } & 8 May 1997 & 65 & -148 & $18-32$ & 1 profile & \\
\hline & 7 July 1997 & 65 & -148 & $18-30$ & 1 profile & \\
\hline Kiruna, Sweden & 3 Dec 1999 & 68 & 21 & $18-32$ & 1 profile & \\
\hline POLARIS I & 26 April 1997 & $81-88$ & $\mathrm{n} / \mathrm{a}$ & $17-19$ & 4 & \multirow[t]{13}{*}{ Park et al. (2004) } \\
\hline \multirow[t]{4}{*}{ POLARIS II } & 29 June 1997 & 62 & $\mathrm{n} / \mathrm{a}$ & 21 & 1 & \\
\hline & 30 June 1997 & $63-66$ & $\mathrm{n} / \mathrm{a}$ & $19-21$ & 3 & \\
\hline & 7 July 1997 & $73-89$ & $\mathrm{n} / \mathrm{a}$ & $17-21$ & 3 & \\
\hline & 10 July 1997 & 64 & $\mathrm{n} / \mathrm{a}$ & 21 & 1 & \\
\hline \multirow[t]{2}{*}{ POLARIS III } & 15 Sep 1997 & 65 & $\mathrm{n} / \mathrm{a}$ & $13-19$ & 4 & \\
\hline & 18 Sep 1997 & 79 & $\mathrm{n} / \mathrm{a}$ & 19 & 1 & \\
\hline \multirow[t]{6}{*}{ SOLVE } & 23 Jan 2000 & $63-65$ & $\mathrm{n} / \mathrm{a}$ & $11-18$ & 3 & \\
\hline & 27 Jan 2000 & $63-66$ & $\mathrm{n} / \mathrm{a}$ & 20 & 3 & \\
\hline & 2 Feb 2000 & 64 & $\mathrm{n} / \mathrm{a}$ & 19 & 1 & \\
\hline & 3 Feb 2000 & 69 & $\mathrm{n} / \mathrm{a}$ & 18 & 1 & \\
\hline & 5 March 2000 & $68-70$ & $\mathrm{n} / \mathrm{a}$ & $17-19$ & 2 & \\
\hline & 11 March 2000 & $61-72$ & $\mathrm{n} / \mathrm{a}$ & $17-20$ & 5 & \\
\hline \multirow[t]{5}{*}{ Sanriku, Japan } & 4 June 1990 & 39 & 142 & $16-35$ & 9 & \multirow{7}{*}{$\begin{array}{l}\text { Toyoda et al. (2001, 2004), } \\
\text { Yoshida and Toyoda (2000) }\end{array}$} \\
\hline & 3 Sep 1998 & 39 & 142 & $15-30$ & 11 & \\
\hline & 31 May 1999 & 39 & 142 & $15-35$ & 11 & \\
\hline & 28 Aug 2000 & 39 & 142 & $15-32$ & 10 & \\
\hline & 30 May 2001 & 39 & 142 & $15-34$ & 11 & \\
\hline Kiruna, Sweden & 22 Feb 1997 & 68 & 20 & $10-26$ & 11 & \\
\hline Syowa, Antarctica & 3 Jan 1998 & -69 & 40 & $10-30$ & 9 & \\
\hline \multirow[t]{2}{*}{ Hyderabad, India } & 26 March 1987 & 18 & 79 & $17-26$ & 5 & \multirow[t]{19}{*}{ Röckmann et al. (2001); this work } \\
\hline & 29 April 1999 & 18 & 79 & $10-28$ & 10 & \\
\hline Aire sur l'Adour & 30 Sep 1993 & 44 & 0 & $10-32$ & 14 & \\
\hline \multirow[t]{2}{*}{ (ASA), France } & 9 Oct 2001 & 44 & -1 & $12-31$ & 14 & \\
\hline & 24 Sep 2002 & 44 & $0-1$ & $12-32$ & 9 & \\
\hline Gap, France & 23 June 1999 & 44 & $3-6$ & $8-34$ & 15 & \\
\hline \multirow[t]{6}{*}{ Kiruna, Sweden } & 18 Jan 1992 & 68 & 21 & $6-27$ & 2 & \\
\hline & 6 Feb 1992 & 68 & 21 & $7-23$ & 9 & \\
\hline & 20 March 1992 & 68 & 21 & $7-25$ & 9 & \\
\hline & 7 March 1995 & 68 & 21 & $7-30$ & 11 & \\
\hline & 1 March 2000 & 68 & $24-28$ & $9-21$ & 11 & \\
\hline & 6 March 2003 & 68 & $22-27$ & $11-30$ & 13 & \\
\hline \multirow[t]{7}{*}{ EUPLEX } & 19 Jan 2003 & 77 & 21 & 20 & 1 & \\
\hline & 23 Jan 2003 & $66-73$ & $16-18$ & $18-20$ & 13 & \\
\hline & 26 Jan 2003 & $66-73$ & $21-23$ & $18-19$ & 5 & \\
\hline & 6 Feb 2003 & $66-79$ & $9-19$ & $17-20$ & 16 & \\
\hline & 8 Feb 2003 & $67-72$ & $15-27$ & $17-20$ & 18 & \\
\hline & 9 Feb 2003 & $68-80$ & $23-49$ & $14-19$ & 19 & \\
\hline & 11 Feb 2003 & $67-70$ & $12-26$ & $8-15$ & 16 & \\
\hline
\end{tabular}




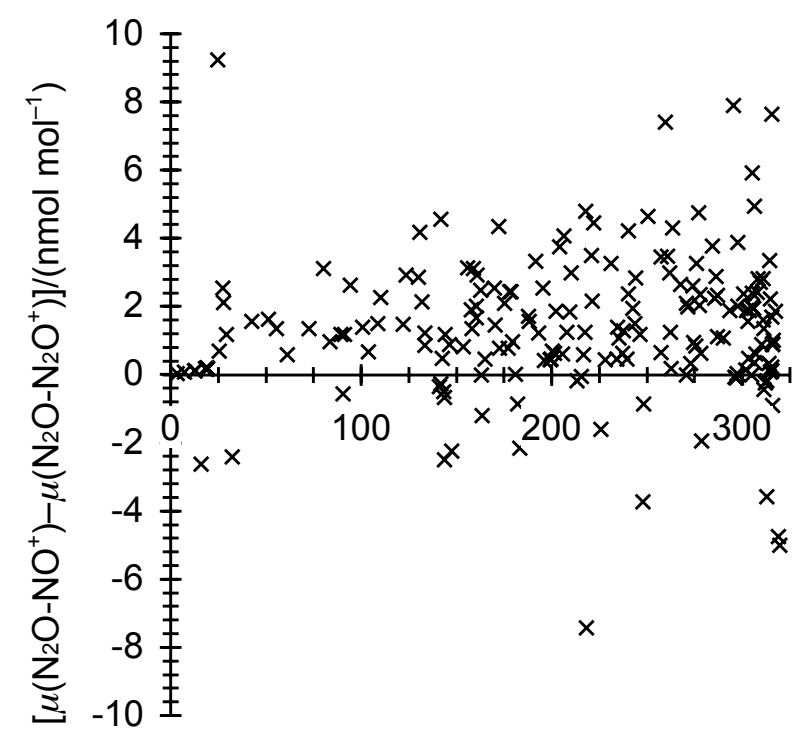

$\mu\left(\mathrm{N}_{2} \mathrm{O}-\mathrm{N}_{2} \mathrm{O}^{+}\right) /\left(\mathrm{nmol} \mathrm{mol}^{-1}\right)$

Fig. 1. Difference between $\mathrm{N}_{2} \mathrm{O}$ mixing ratios derived from the $\mathrm{NO}^{+}$fragment ion and the $\mathrm{N}_{2} \mathrm{O}^{+}$molecular ion peak areas versus $\mathrm{N}_{2} \mathrm{O}$ mixing ratios derived from the $\mathrm{N}_{2} \mathrm{O}^{+}$molecular ion peak area (195 of 213 samples). The average difference is $(1.3 \pm 2.1) \mathrm{nmol} \mathrm{mol}^{-1}$.

$\delta^{15} \mathrm{~N}$ and $\delta^{18} \mathrm{O}$ and about $0.5 \%$ of ${ }^{1} \delta^{15} \mathrm{~N}$ and ${ }^{2} \delta^{15} \mathrm{~N}$, which is the best precision achieved to date for stratospheric $\mathrm{N}_{2} \mathrm{O}$ samples (Park et al., 2004; Toyoda et al., 2004).

Mixing ratios $(\mu)$ were calculated by comparing the extraction time-weighted peak areas of the stratospheric samples with those of the Schauinsland reference tank samples. The $\mathrm{N}_{2} \mathrm{O}$ mixing ratio of the Schauinsland reference tank was determined by Ingeborg Levin at the Institute for Environmental Physics, Heidelberg/Germany, using GC-ECD (gas chromatography-electron capture detection) and found to be $(319.0 \pm 0.2) \mathrm{nmol} / \mathrm{mol}$ (SIO98 scale; Prinn et al., 2000). $\mathrm{N}_{2} \mathrm{O}$ mixing ratios were derived both from $\mathrm{NO}^{+}$fragment and $\mathrm{N}_{2} \mathrm{O}^{+}$molecular ion peak areas. Fig. 1 shows that they follow a 1:1 relationship very closely, with the $\mathrm{NO}^{+}$derived mixing ratio being on average $(1.3 \pm 2.1) \mathrm{nmol} \mathrm{mol}^{-1}$ higher. The average relative difference, which is more important for the derivation of stratospheric apparent fractionation constants, is $(0.7 \pm 1.1) \%$ (excluding five outliers of $-15 \%$, $-7 \%, 8 \%, 10 \%$, and $46 \%$ ). We adopt the mean of the $\mathrm{N}_{2} \mathrm{O}^{+}$and the $\mathrm{NO}^{+}$-derived mixing ratio as the mixing ratio of the individual sample. For a subset of 47 samples, we have compared this mixing ratio to independent GC-ECD measurements at the Institute for Meteorology and Geophysics of the University of Frankfurt (Fig. 2). The average difference between these two values is $-(0.2 \pm 2.4) \mathrm{nmol} \mathrm{mol}^{-1}$, and the average relative difference is $(-0.3 \pm 2.1) \%$ (excluding two outliers of $-18 \%$ and $11 \%$ ), with relative differences of $1 \%$

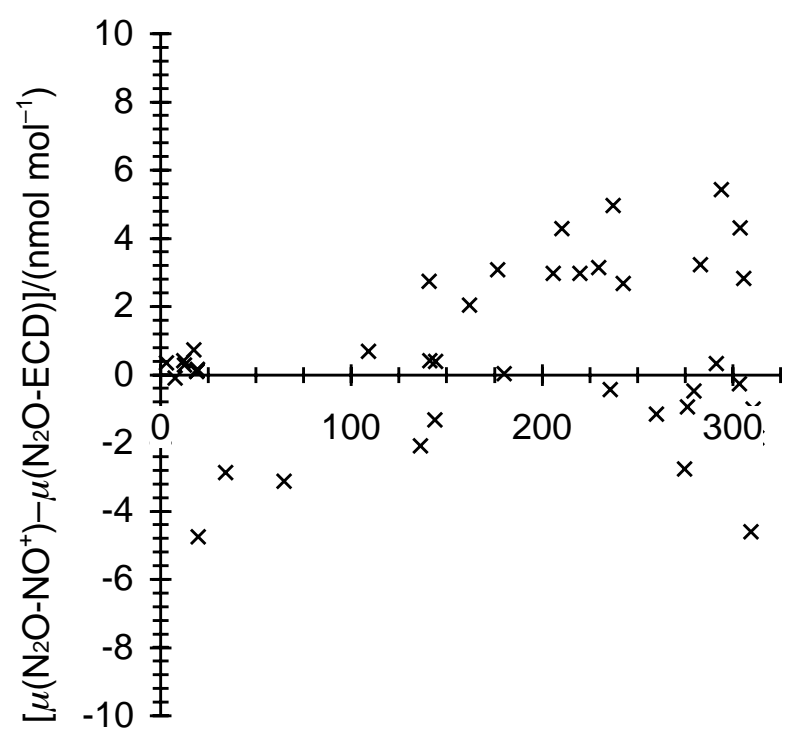

$\mu\left(\mathrm{N}_{2} \mathrm{O}-\mathrm{ECD}\right) /\left(\mathrm{nmol} \mathrm{mol}{ }^{-1}\right)$

Fig. 2. Difference between $\mathrm{N}_{2} \mathrm{O}$ mixing ratio determined with mass-spectrometric (MS) and electron capture detection (ECD) versus $\mathrm{N}_{2} \mathrm{O}$ mixing ratio determined by ECD ( 47 of 213 samples). The average difference is $-(0.2 \pm 2.4) \mathrm{nmol} \mathrm{mol}^{-1}$.

or smaller for larger mixing ratios and larger relative differences for smaller mixing ratios. Thus, precision and accuracy of the mixing ratio measurements by mass spectrometry are sufficient for the derivation of apparent fractionation constants with an uncertainty of about $1 \%$.

\section{Results and discussion}

We start this section with a general description of the balloon profiles in terms of their mixing ratios and then proceed to explore the variation of the isotopic composition with the mixing ratio. In a first step, we will interpret the data in a Rayleigh fractionation framework, as applied in past studies of stratospheric $\mathrm{N}_{2} \mathrm{O}$ (Griffith et al., 2000; Park et al., 2004; Rahn and Wahlen, 1997; Röckmann et al., 2001; Toyoda et al., 2001). As a second step and partly in response to a question raised by Park et al. (2004), we explore to what extent simple two-end-member mixing can describe the covariation between isotope and mixing ratios and whether a more complex "continuous weak mixing" scheme (Plumb et al., 2000) can give a better description for part of the data. Finally, we investigate whether correlations between $\mathrm{N}_{2} \mathrm{O}$ isotope signatures are useful indicators of the partitioning between $\mathrm{O}\left({ }^{1} \mathrm{D}\right)$ and photolytic $\mathrm{N}_{2} \mathrm{O}$ sinks and of changes of temperature and actinic fluxes with altitude. 


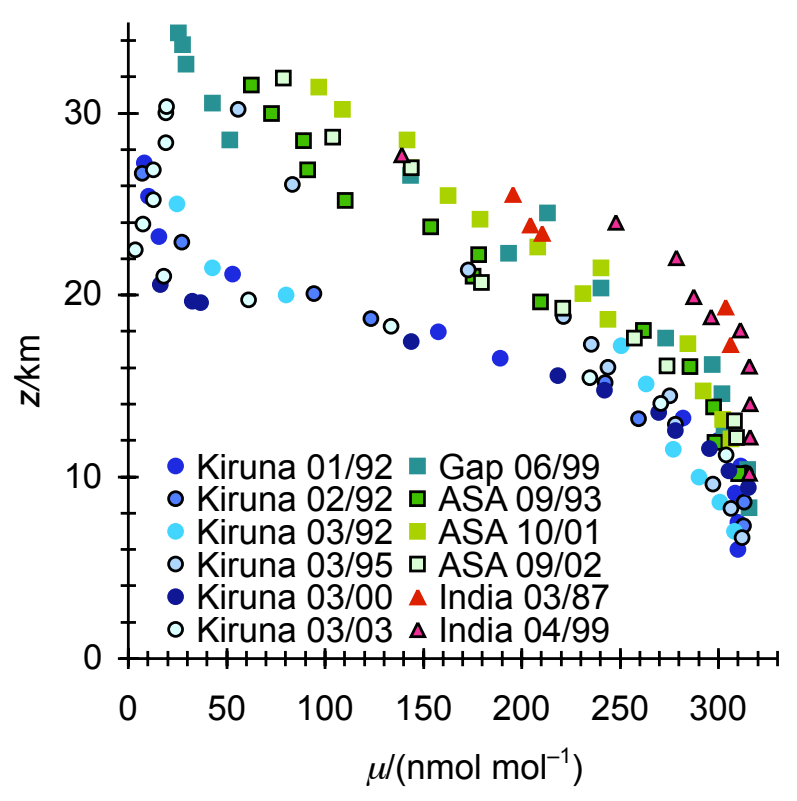

Fig. 3. Vertical profiles of $\mathrm{N}_{2} \mathrm{O}$ mixing ratios from the balloon flights (ASA: Aire sur l'Adour). The EUPLEX 2003 aircraft samples have been omitted to avoid congestion of the plot. The mixing ratios have not been adjusted for the sampling date and the stratospheric age of air (Sect. 3.2).

\section{$3.1 \quad \mathrm{~N}_{2} \mathrm{O}$ mixing ratios}

Figure 3 shows vertical profiles of the $\mathrm{N}_{2} \mathrm{O}$ mixing ratio. The decrease of tropopause altitude, $z_{\text {trop }}$, from the low-latitude Indian samples $\left(z_{\text {trop }} \approx 16 \mathrm{~km}\right)$ to the polar Kiruna samples $\left(z_{\text {trop }} \approx 7 \mathrm{~km}\right)$ can be estimated from the point where the mixing ratio starts to drop below its tropospheric value of 310 to $320 \mathrm{nmol} \mathrm{mol}^{-1}$. Correspondingly, the profiles can be separated into those of polar character (all Kiruna profiles except for the high altitude-samples of the Kiruna 03/95 profile), mid-latitude character (Gap 06/99, all Aire sur l'Adour profiles, and the high-altitude Kiruna 03/95 samples) and tropical character (India profiles). The Kiruna profiles were obtained in winter and generally sampled air from inside the polar vortex, although the distinction between polar and midlatitude samples is only unambiguous for mixing ratios below $290 \mathrm{nmol} \mathrm{mol}^{-1}$. In contrast, the polar vortex had already broken up completely by the time of the 03/95 balloon launch and the resulting $\mathrm{N}_{2} \mathrm{O}$ profile corresponds to a mid-latitude one. The distinction between tropical and midlatitude samples can be made most clearly for mixing ratios above $250 \mathrm{nmol} \mathrm{mol}^{-1}$, but some overlap occurs for lower mixing ratios.

The above classification based on the vertical $\mathrm{N}_{2} \mathrm{O}$ profiles is rather crude and sensitive to intra- and interannual synoptic changes of the large-scale stratospheric circulation. Correlations between different stratospheric trace gases are better suited to segregate the stratosphere into regions be- cause characteristic tracer-tracer relationships develop in areas where exchange time-scales due to transport and mixing are significantly shorter than the instantaneous chemical lifetimes. Michelsen et al. (1998) developed an $\mathrm{N}_{2} \mathrm{O}-\mathrm{CH}_{4}$ climatology with polynomial fits for different stratospheric regions and we show our data in context of these fits in Fig. 4. No clear separation into stratospheric regions is possible for $\mathrm{N}_{2} \mathrm{O}$ mixing ratios above $150 \mathrm{nmol} \mathrm{mol}^{-1}$, but below this value, the picture that developed from the vertical $\mathrm{N}_{2} \mathrm{O}$ profiles is confirmed with one exception: The $140 \mathrm{nmol} \mathrm{mol}^{-1}$ sample obtained from the India 04/99 launch clearly stands out as a tropical $\mathrm{N}_{2} \mathrm{O}$ sample, whereas the India 03/87 samples rather show mid-latitude character. This is in line with historic $\mathrm{CH}_{4}$ profile variations, which showed that the intertropical convergence zone (ITCZ) moves northward across the sampling location of Hyderabad between end of March and end of April, but was still south of Hyderabad on 26 March 1987 (Patra et al., 2003). Some caution is warranted in the interpretation of our data in context of these $\mathrm{CH}_{4}-\mathrm{N}_{2} \mathrm{O}$ relationships because the latter have been derived with data from the years 1993 and 1994 (Michelsen et al., 1998), but will change in time due to the different relative growth rates of atmospheric $\mathrm{N}_{2} \mathrm{O}$ and $\mathrm{CH}_{4}$ of $0.25 \% / \mathrm{a}$ and between 0 and $0.8 \% / \mathrm{a}$, respectively (Prather et al., 2001). This, in addition to natural variability, may explain why some samples fall above or below the generic $\mathrm{N}_{2} \mathrm{O}-\mathrm{CH}_{4}$ climatology.

3.2 Isotope variations in a Rayleigh fractionation framework

Figure 5 shows the relationship between average $\delta^{15} \mathrm{~N}$ values (relative to SIL- $\mathrm{N}_{2} \mathrm{O}$ ) and $\mathrm{N}_{2} \mathrm{O}$ mixing ratios. The isotopic enrichment generally increases with decreasing mixing ratios due to the increasing degree of photochemical removal of $\mathrm{N}_{2} \mathrm{O}$ and the associated kinetic isotope effects, which lead to preferential destruction of the lighter isotopologues. Samples with mixing ratios above $200 \mathrm{nmol} \mathrm{mol}^{-1}$ display a uniform compact relationship independent of latitude, which was also noted by Park et al. (2004). However, samples with $\mu<200 \mathrm{nmol} \mathrm{mol}^{-1}$ clearly split up into different profiles, depending on latitude and sampling season. Mid-latitude samples collected in fall maintain a rather compact relationship even below $200 \mathrm{nmol} \mathrm{mol}^{-1}$. In contrast, samples from polar latitudes (Kiruna) and the mid-latitude summer profile (Gap 06/99) differ substantially among each other and from the fall mid-latitude profiles. Notably, the Kiruna $03 / 95$ profile, which was classified as a mid-latitude profile based on its $\mathrm{CH}_{4}-\mathrm{N}_{2} \mathrm{O}$ correlation and the shape of the vertical $\mathrm{N}_{2} \mathrm{O}$ profile, falls below the ASA fall profiles. It is similar to the Gap 06/99 summer profile, though. The polar vortex samples show $\delta^{15} \mathrm{~N}$ variations of up to about $30 \%$ of the same $\mathrm{N}_{2} \mathrm{O}$ mixing ratio, which means that the photochemical and transport processes that establish the mid-latitude $\delta$ - $\mu$ relationship do not prevail in the polar vortex. This is due to dynamic isolation of the polar vortex from mid-latitude air 


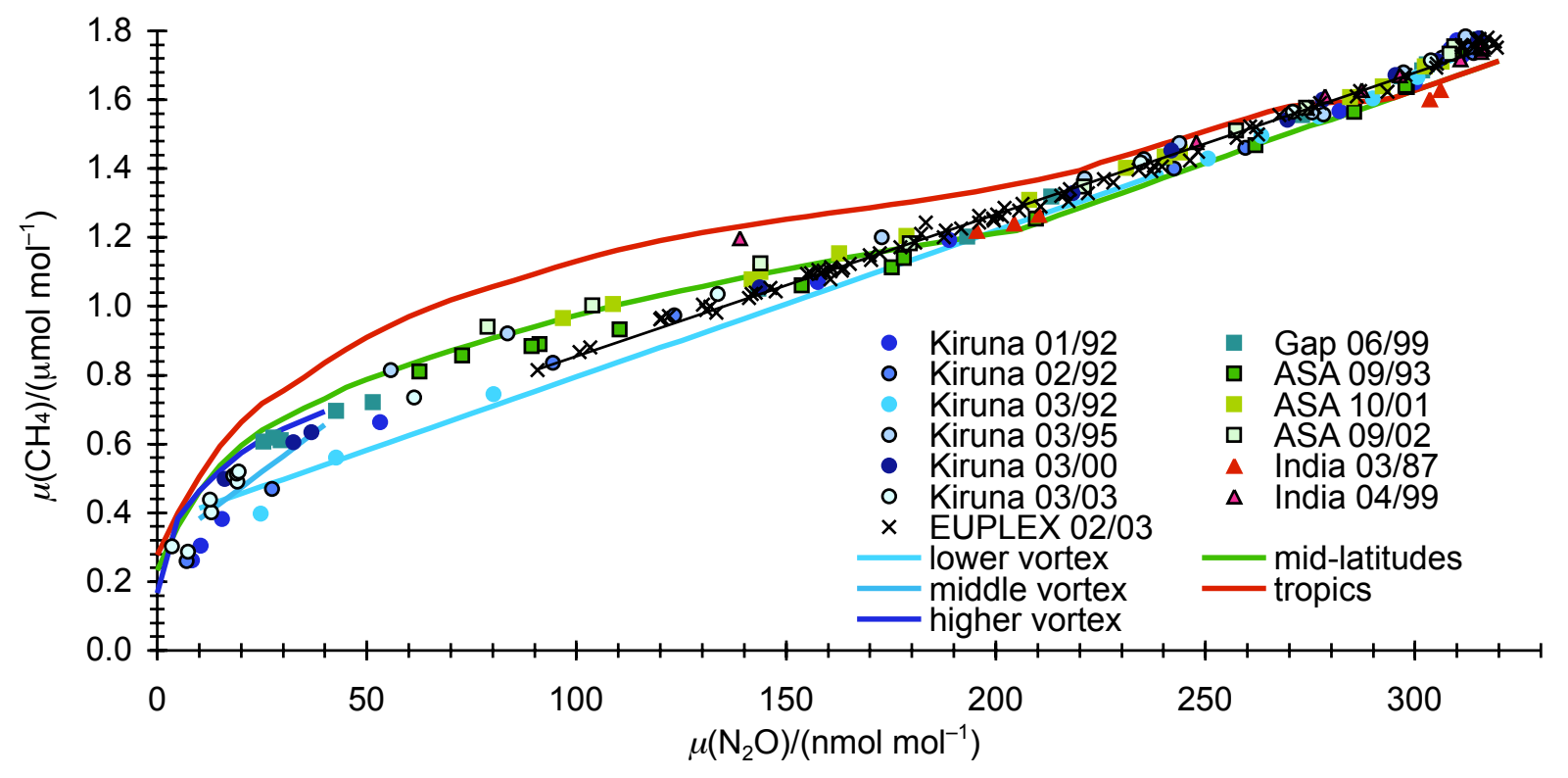

Fig. 4. Correlation between $\mathrm{CH}_{4}$ and $\mathrm{N}_{2} \mathrm{O}$ mixing ratios, measured by gas chromatography-isotope ratio mass spectrometry. Reference curves for vortex, mid-latitude and tropical air are from ATMOS measurements (Michelsen et al., 1998).

masses and the limited degree of photochemical processing that occurs over the dark polar areas. In addition, mixing with upper stratospheric and/or mesospheric air (Sect. 3.3) can play a role.

For illustrative purposes, Fig. 5 also shows two hypothetical Rayleigh fractionation lines, which have been calculated assuming a closed system with no sources and a single sink with an isotope effect $\varepsilon$ of $-38.0 \%$ and $-19.2 \%$, respectively. Rayleigh fractionation then leads to $\delta=\left(\mu / \mu_{\mathrm{T}}\right)^{\varepsilon}-1$, with $\mu / \mu_{\mathrm{T}}$ being the $\mathrm{N}_{2} \mathrm{O}$ mixing ratio at the tropopause. The two limits for $\varepsilon$ correspond to an intrinsic photochemical isotope effect as expected from broadband photolysis at room temperature with a $10 \%$ contribution from $\mathrm{N}_{2} \mathrm{O}$ photo-oxidation and the apparent isotope effect expected for transport-limited conditions (about half the intrinsic isotopic effect) (Kaiser et al., 2002a, b). These upper and lower bounds delimit the range of isotope effects, which can be realized in a purely one-dimensional reaction-advectiondiffusion system at steady-state (see also Sect. 3.5). This was demonstrated for the reaction-diffusion system (Kaiser et al., 2002a; Kaye, 1987; Morgan et al., 2004), but is still valid even if advection is included due to the linearity of the corresponding differential equation (Kaiser and Röckmann, $2006^{1}$ ). As noted in most previous publications on stratospheric $\mathrm{N}_{2} \mathrm{O}$ isotopes (e.g., Rahn et al., 1998; Röckmann et al., 2001; Toyoda et al., 2001), the stratospheric measurements clearly fall below the $\delta-\mu$ relationship defined by the intrinsic isotope effect, which is due to transport and mixing

\footnotetext{
${ }^{1}$ Kaiser, J. and Röckmann, T.: Effective isotope effects in atmospheric and oceanic environments, in preparation, 2006.
}

effects. The magnitude of the intrinsic isotope effect used here is only an estimate because, on the one hand, the photolytic isotope effect is larger at lower (stratospheric) temperatures (Kaiser et al., 2002b; von Hessberg et al., 2004) and, on the other hand, the contribution of the photo-oxidation sink may vary (Sect. 3.5). In any case, a simple Rayleigh model fails to describe the measurements. For mixing ratios below $70 \mathrm{nmol} \mathrm{mol}^{-1}$, the attenuation of the intrinsic isotope effect due to diffusion and advection can explain this discrepancy because the observations lie mostly above the apparent fractionation constant under transport-limited conditions. However, at mixing ratios greater than $70 \mathrm{nmol} \mathrm{mol}^{-1}$, some $\delta$ values are even smaller than expected for the onedimensional, transport-limited case. This means that other effects such as mixing have to be invoked to explain the observations (see Sect. 3.3). It should also be noted that the above analysis is simplified in the sense that it uses only tropospheric $\mathrm{N}_{2} \mathrm{O}$ mixing ratios and $\delta$ values as initial conditions for the Rayleigh fractionation approximation and does not consider variations of the fractionation constant as $\mathrm{N}_{2} \mathrm{O}$ gets depleted. A more appropriate approach will therefore be used in the following section, in which we use the local slope in a Rayleigh fractionation plot to estimate the apparent fractionation constant.

3.2.1 Normalization for atmospheric trends and stratospheric age of air

For a quantitative analysis of the observed $\delta$ values in a Rayleigh fractionation framework, we apply a normalization for the time of sampling (the samples span a range of 16 


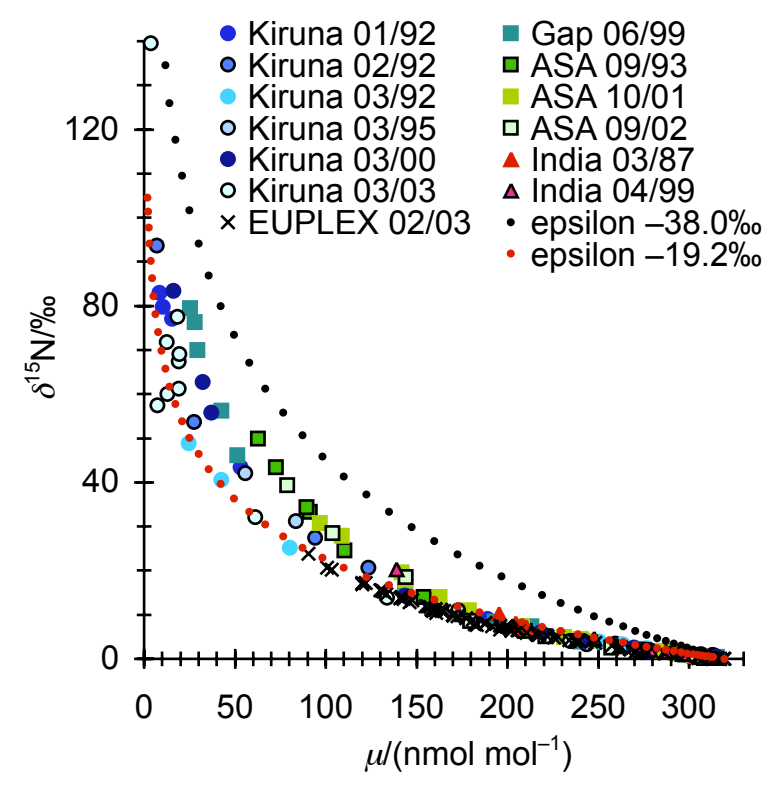

Fig. 5. Compact relationship between $\delta$ values and mixing ratios down to $200 \mathrm{nmol} \mathrm{mol}^{-1}$, divergent relationship below, with higher $\delta$ values for mid-latitude samples than for polar locations. However, note the mid-latitude profile for Kiruna 03/95 (outside of polar vortex), which was classified as a mid-latitude profile based on its $\mathrm{CH}_{4}-\mathrm{N}_{2} \mathrm{O}$ correlation (Sect. 3.2 and Fig. 4), but is within the range of other polar profiles in $\delta$ - $\mu$ space. $\delta$ values are relative to SIL$\mathrm{N}_{2} \mathrm{O}$. Also shown are two hypothetical Rayleigh fractionation lines, which have been calculated assuming isotope effects $\varepsilon$ of $-38.0 \%$ and $-19.2 \%$, respectively. These two limits for $\varepsilon$ correspond to an intrinsic photochemical isotope effect as expected from broadband photolysis at room temperature with a $10 \%$ contribution from $\mathrm{N}_{2} \mathrm{O}$ photo-oxidation and the apparent isotope effect expected for transport-limited conditions (about half the intrinsic isotopic effect) (Kaiser et al., 2002a, b).

years) and the age of stratospheric air. In order to make the different profiles intercomparable, we first adjust the measured isotopic compositions to a specific date in order to normalize for the changing isotopic composition of modern atmospheric $\mathrm{N}_{2} \mathrm{O}$. Due to anthropogenic inputs of isotopically light $\mathrm{N}_{2} \mathrm{O}$, the $\delta$ values are decreasing with time, as deduced from analyses of Antarctic firn air (Bernard et al., 2006; Röckmann et al., 2003a; Sowers et al., 2002) and direct atmospheric measurements (Röckmann and Levin, 2005). As a base date, we arbitrarily chose the sampling date of the SIL- $\mathrm{N}_{2} \mathrm{O}$ reference tank (15 March 2002). The normalization correction we apply is based on recent direct measurements of the position-dependent isotope composition of archived, clean southern hemisphere background air samples collected at the German Antarctic "Georg von Neumayer" Station $\left(70^{\circ} 39^{\prime} \mathrm{S}, 08^{\circ} 15^{\prime} \mathrm{W}\right)$. Röckmann and Levin (2005) found linear trends of $(-0.040 \pm 0.003) \%$ ola for $\delta^{15} \mathrm{~N},(-0.064 \pm 0.016) \%$ o a for ${ }^{1} \delta^{15} \mathrm{~N},(-0.014 \pm 0.016) \% o / \mathrm{a}$ ${ }^{2} \delta^{15} \mathrm{~N}$, and $(-0.021 \pm 0.003) \% o / \mathrm{a}$ for $\delta^{18} \mathrm{O}$ over the time-span of available samples (between March 1990 and November 2002). We assume that these linear trends were constant over the time-span defined by the stratospheric samples (March 1987 to March 2003).

In order to investigate the influence of transport and chemistry on the isotopic composition of stratospheric $\mathrm{N}_{2} \mathrm{O}$, we linearize our data in a Rayleigh fractionation plot of $\ln (1+\delta)$ vs. $\ln \left(\mu / \mu_{\mathrm{T}}\right)$ (The error due to using $\mathrm{N}_{2} \mathrm{O}$ rather than ${ }^{14} \mathrm{~N}_{2}^{16} \mathrm{O}$ mixing ratios can be neglected; see Kaiser et al., 2002a). The local slope then corresponds to an apparent fractionation constant. To pursue this approach as accurately as possible, we have to estimate $\mu_{\mathrm{T}}$, the $\mathrm{N}_{2} \mathrm{O}$ mixing ratio at the tropopause when the air parcel entered the stratosphere, and apply a further normalization correction to $\delta$ to take the age of air into account. These normalization corrections are small relative to the measurement error (usually $<1 \%$ ), but were deemed necessary in order to achieve a closer analogy to a Rayleigh fractionation system. Therefore, we consider the age of stratospheric air in order to normalize all data to a single stratospheric entry datum. The age of air is defined as the time elapsed since an air parcel has passed the tropopause. We base our age-ofair estimate on the relationship between $\mathrm{N}_{2} \mathrm{O}$ mixing ratios and the age of mid-latitude/lower polar vortex air found by Boering et al. (1996) for samples from the years 1992 to 1996. A polynomial regression of the age of air $\Gamma$ versus $\mu / \mu_{\mathrm{T}}$ gives $\Gamma\left(\mu / \mu_{\mathrm{T}}\right) / \mathrm{a}=-(7.43 \pm 0.34)\left(\mu / \mu_{\mathrm{T}}\right)^{3}+(3.68 \pm 0.56)$ $\left(\mu / \mu_{\mathrm{T}}\right)^{2}-(1.94 \pm 0.28) \mu / \mu_{\mathrm{T}}+5.69 \pm 0.04\left(R^{2}=0.998\right)$, with the tropopause mixing ratio $\mu_{\mathrm{T}}$ adjusted to $311.6 \mathrm{nmol} \mathrm{mol}^{-1}$ to give an age of zero for $\mu=\mu_{\mathrm{T}}$. The value of $311.6 \mathrm{nmol} \mathrm{mol}^{-1}$ agrees with AGAGE observations at the northern hemisphere background station Mace Head/Ireland in 1994. However, given the time range of observations (1992 to 1996), the global extent of sampling locations used by Boering et al. (1996) and the age difference of tropopause air relative to tropospheric air, one might have expected a slightly lower value than $311.6 \mathrm{nmol} \mathrm{mol}^{-1}$. The difference might be due to different $\mathrm{N}_{2} \mathrm{O}$ calibration scales. The use of $\mu / \mu_{\mathrm{T}}$ for the $\Gamma$ parameterization rather than $\mu$ itself minimizes errors due to the increase of the $\mathrm{N}_{2} \mathrm{O}$ mixing ratio with time. The parameterization will differ slightly for polar and tropical samples, but the differences only lead to secondorder corrections for the present application and can be neglected. Engel et al. (2002) have derived a similar third-order polynomial regression of $\Gamma$ versus the $\mathrm{N}_{2} \mathrm{O}$ mixing ratio. The age difference between the parameterization of Engel et al. (2002) and that based on the data of Boering et al. (1996) is at most 4 months, which can be neglected for the present application.

We use the estimated age of air and assume that the age difference between tropopause and tropospheric air is 0.8 years (Volk et al., 1997). We then calculate the isotopic composition of the stratospheric sample relative to its composition when it left the tropopause, using the same isotope trends as above (Röckmann and Levin, 2005). Similarly, 
we estimate the tropopause $\mathrm{N}_{2} \mathrm{O}$ mixing ratio for individual samples. The northern-hemisphere AGAGE data from Mace Head (Ireland) are used as reference for the normalization, because all our data are from the northern hemisphere. Second-order effects due to intrahemispheric $\mathrm{N}_{2} \mathrm{O}$ mixing ratio gradients are neglected. For the pre-AGAGE period before 1994, we use the GAGE data from Cape Grim (Australia) instead because they display more consistent interannual variations and a cleaner seasonal signal than the GAGE data from Mace Head record. In order to account for interhemispheric $\mathrm{N}_{2} \mathrm{O}$ mixing ratio differences, the Cape Grim data are time shifted forward by 0.8 months and adjusted upward by $0.7 \mathrm{nmol} \mathrm{mol}^{-1}$. This adjustment is based on a comparison between the AGAGE data from Cape Grim and Mace Head for the years 1994 to 2002.

\subsubsection{Calculation of $\varepsilon_{\text {app }}$ from global fits and local slopes}

Using the normalized $\mathrm{N}_{2} \mathrm{O}$ isotope and mixing ratios, apparent fractionation constants $\left(\varepsilon_{\text {app }}\right)$ are calculated from $\ln (1+\delta)$ and $\ln \left(\mu / \mu_{\mathrm{T}}\right)$ (Fig. 6). We use two different approaches to do this. First, we calculate average linear least-squares $\varepsilon_{\text {app }}$ values for individual lower stratosphere profiles using only samples with $\ln \left(\mu / \mu_{\mathrm{T}}\right)>-0.6$. An $\ln \left(\mu / \mu_{\mathrm{T}}\right)$ value of -0.6 corresponds to $\mathrm{N}_{2} \mathrm{O}$ mixing ratios between 168 and $174 \mathrm{nmol} \mathrm{mol}^{-1}$ for the age range of our samples. The same cut-off has already been used by Kaiser (2002) and von Hessberg et al. (2004) and corresponds to the region of the Rayleigh fractionation plot without noticeable curvature, defined by $0>\ln \left(\mu / \mu_{\mathrm{T}}\right)>-0.6$. For $\ln \left(\mu / \mu_{\mathrm{T}}\right)<-0.6$, the Rayleigh fractionation plots show positive curvature and a linear fit is therefore no longer appropriate. Instead, we fit a second-order polynomial to the individual profiles and calculate local slopes from the first derivative of the fit at exemplary $\ln \left(\mu / \mu_{\mathrm{T}}\right)$ values of $-1.0,-1.5$ and -2.0 . This serves to illustrate how $\varepsilon_{\text {app }}$ values change for higher altitude samples. We exclude samples with $\ln \left(\mu / \mu_{\mathrm{T}}\right)<-2.4$ from the fit because these samples are influenced by mixing with low- $\mathrm{N}_{2} \mathrm{O}$ upper stratospheric and mesospheric air (Sect. 3.3). Compared to previous studies, in which linear fits were applied to both the middle and the lower stratospheric samples (Kaiser, 2002; Park et al., 2004; Toyoda et al., 2001, 2004), our present approach has the advantage to expose differences between individual profiles more clearly and to decrease the influence of the most enriched samples on the overall fit. For illustration, the average regression coefficient $R^{2}$ for all ${ }^{15} \mathrm{~N}$ Rayleigh plots improves from 0.990 to 0.997 for linear and quadratic fits of samples with $\left.\ln \left(\mu / \mu_{\mathrm{T}}\right)>-0.6\right)$, from 0.997 to 0.998 for linear and quadratic fits of samples with $\left.\ln \left(\mu / \mu_{\mathrm{T}}\right)<-0.6\right)$ and from 0.987 to 0.999 for linear and quadratic fits of all samples. Higher-fitting orders do not lead to a signficant improvement of the goodness-of-fit.

A comparison of the $\varepsilon_{\text {app }}$ values derived from the normalized $\mathrm{N}_{2} \mathrm{O}$ isotope and mixing ratios with $\varepsilon_{\text {app }}$ values derived from the non-normalized data shows differences be- tween -0.4 and $+0.2 \%$ for $\varepsilon^{15} \mathrm{~N}$. The normalizations described in Sect. 3.2.1 are therefore of minor importance for the $\varepsilon_{\text {app }}$ values, but the isotope ratio normalization turn out to be significant for the study of correlations between different intramolecular isotope signatures in the lower stratosphere (Sect. 3.5).

\subsubsection{Vertical and meridional trends in $\varepsilon_{\mathrm{app}}$}

The apparent Rayleigh fractionation constants we derive according to the two approaches described above are shown in Table 2 and, for $\varepsilon^{15} \mathrm{~N}$, in Fig. 7 as a function of latitude. The trends are qualitatively the same for other $\mathrm{N}_{2} \mathrm{O}$ isotope signatures due to their high correlation (see Sect. 3.5).

In general, the absolute magnitude of $\varepsilon_{\text {app }}$ decreases with increasing distance from the equator. The latter relationship is what one would expect qualitatively for faster vertical transport time-scales near the equator (photochemistry is rate-limiting). This effect outweighs the faster photolysis rates due to higher actinic fluxes at low latitudes.

$\left|\varepsilon_{\text {app }}\right|$ increases with altitude, but even at middle stratospheric altitudes $\left(\ln \left(\mu / \mu_{\mathrm{T}}\right)=-2.0\right),\left|\varepsilon^{15} \mathrm{~N}_{\mathrm{app}}\right|$ values remains below the expected absolute magnitude of the intrinsic fractionation constant of about $46 \%$ o. The latter estimate is derived from the photolytic fractionation constant of about $-51 \%$ o, computed from absorption cross section measurements (von Hessberg et al., 2004) for a representative stratospheric temperature of $233 \mathrm{~K}$ and actinic fluxes corresponding to altitudes between 20 and $30 \mathrm{~km}$ (Minschwaner et al., 1993), and the fractionation constant of $-5.5 \%$ ofor the $\mathrm{N}_{2} \mathrm{O}$ $+\mathrm{O}\left({ }^{1} \mathrm{D}\right)$ reaction (Kaiser et al., 2002a), with a $10 \%$ contribution of the $\mathrm{N}_{2} \mathrm{O}+\mathrm{O}\left({ }^{1} \mathrm{D}\right)$ reaction to the total $\mathrm{N}_{2} \mathrm{O}$ sink. Broadband photolysis measurements with a lamp simulating stratospheric ultraviolet light conditions (Röckmann et al., 2001) give a similar value for the photolytic fractionation constant at $233 \mathrm{~K}$, namely $-48 \%$ (Kaiser et al., 2002b).

At $\ln \left(\mu / \mu_{\mathrm{T}}\right)=-2.0,\left|\varepsilon_{\mathrm{app}}\right|$ is usually above half the absolute magnitude of the intrinsic fractionation constant, in line with simulated values based on vertical eddy diffusion coefficients and $\mathrm{N}_{2} \mathrm{O}$ destruction rates (Kaiser, 2002; Kaiser et al., 2002a; Toyoda et al., 2004). However, even though the apparent fraction constants could possibly be reconciled with certain diffusion coefficients and destruction rates, the increase of the magnitude of the apparent fractionation constant with altitude is actually opposite to what one would expect from a simple vertical reaction-diffusion-advection model (Kaiser, 2002; Toyoda et al., 2004). Despite the conceptual usefulness of this one-dimensional description, it has to be stressed that stratospheric transport cannot be characterized as a function of altitude alone and that vertical diffusion is actually not an important process, even though it can be helpful to describe stratospheric transport to some extent. Meridional transport schemes have to be included to explain the variation of $\varepsilon_{\text {app }}$. Possible schemes based on mixing processes are discussed in Sect. 3.3. 

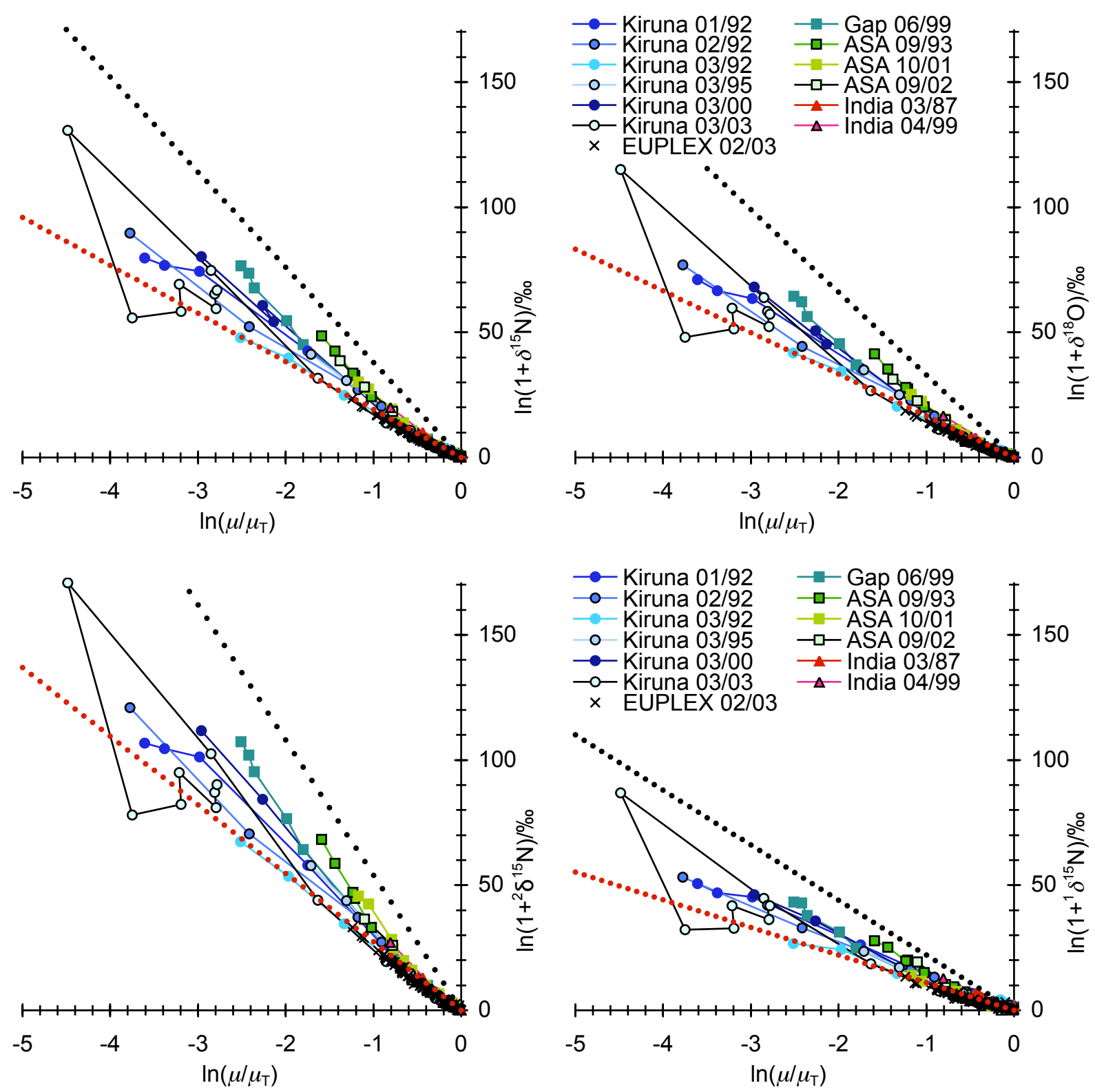

Fig. 6. Rayleigh plots for bulk nitrogen, oxygen and position-dependent nitrogen isotope signatures of stratospheric $\mathrm{N}_{2} \mathrm{O}$. The $\delta$ values are expressed relative to the isotopic composition of tropopause $\mathrm{N}_{2} \mathrm{O}$ at the time the sample entered the stratosphere, which takes the decreasing heavy isotope content of tropospheric $\mathrm{N}_{2} \mathrm{O}$ and the age of the stratospheric air sample into account. Similarly, the mixing ratio of tropopause $\mathrm{N}_{2} \mathrm{O}$ that we use to calculate $\ln \left(\mu / \mu_{\mathrm{T}}\right)$ is normalized for the increasing atmospheric $\mathrm{N}_{2} \mathrm{O}$ mixing ratio and the age of air (see Sect. 3.2 for details). Black and red dotted lines correspond to Rayleigh fractionation with fractionation constants $\varepsilon^{15} \mathrm{~N}=-38.0 \%$ ol $-19.2 \%$, $\varepsilon^{18} \mathrm{O}=-33 \%$ o/ $-16.6 \%$ o, ${ }^{1} \varepsilon^{15} \mathrm{~N}=-22.0 /-11.1 \%$ o, and ${ }^{2} \varepsilon^{15} \mathrm{~N}=-54.0 \%$ ol-27.4\%o, respectively. 
Table 2. Apparent Rayleigh fractionation constants $\left(\varepsilon_{\text {app }}\right)$ derived for lower stratospheric samples (linear fit for $\left.\ln \left(\mu / \mu_{\mathrm{T}}\right)>-0.6\right)$ and for middle stratospheric samples at $\ln \left(\mu / \mu_{\mathrm{T}}\right)$ values of $-1.0,-1.5$ and -2.0 (first derivative of second order polynomial fit for $\ln \left(\mu / \mu_{\mathrm{T}}\right)>-2.4$ ). Extrapolated values are shown in parentheses. The uncertainties originate from the global fits described in Sect. 3.2.2.

\begin{tabular}{|c|c|c|c|c|c|c|c|c|}
\hline \multirow{3}{*}{$\begin{array}{l}\ln \left(\mu / \mu_{\mathrm{T}}\right) \\
\text { India 03/87 }\end{array}$} & \multicolumn{4}{|c|}{$-\varepsilon^{15} \mathrm{~N}_{\mathrm{app}}$} & \multicolumn{4}{|c|}{$-\varepsilon^{18} \mathrm{O}_{\mathrm{app}}$} \\
\hline & $>-0.6$ & -1.0 & -1.5 & -2.0 & $>-0.6$ & -1.0 & -1.5 & -2.0 \\
\hline & $19 \pm 1$ & & & & $16 \pm 1$ & & & \\
\hline India 04/99 & $18 \pm 1$ & $(27 \pm 1)$ & & & $16 \pm 1$ & $(22 \pm 2)$ & & \\
\hline ASA 09/93 & $17 \pm 0$ & $23 \pm 1$ & $30 \pm 1$ & $(36 \pm 1)$ & $14 \pm 0$ & $19 \pm 1$ & $25 \pm 1$ & $(31 \pm 1)$ \\
\hline ASA $10 / 01$ & $19 \pm 1$ & $25 \pm 2$ & $(30 \pm 3)$ & & $15 \pm 1$ & $20 \pm 2$ & $(25 \pm 2)$ & \\
\hline ASA $09 / 02$ & $16 \pm 0$ & $24 \pm 4$ & $(30 \pm 4)$ & & $12 \pm 0$ & $19 \pm 4$ & $(24 \pm 4)$ & \\
\hline Gap 06/99 & $17 \pm 1$ & $21 \pm 1$ & $24 \pm 1$ & $27 \pm 1$ & $14 \pm 1$ & $17 \pm 1$ & $20 \pm 1$ & $22 \pm 1$ \\
\hline Kiruna $01 / 92$ & $17 \pm 0$ & $20 \pm 0$ & $23 \pm 1$ & $25 \pm 1$ & $14 \pm 0$ & $17 \pm 1$ & $19 \pm 1$ & $21 \pm 1$ \\
\hline Kiruna 02/92 & $14 \pm 0$ & $21 \pm 3$ & $25 \pm 3$ & $28 \pm 4$ & $13 \pm 1$ & $18 \pm 1$ & $21 \pm 1$ & $24 \pm 2$ \\
\hline Kiruna 03/92 & $17 \pm 1$ & $17 \pm 0$ & $18 \pm 1$ & $20 \pm 1$ & $16 \pm 1$ & $14 \pm 1$ & $16 \pm 1$ & $17 \pm 2$ \\
\hline Kiruna 03/95 & $17 \pm 1$ & $21 \pm 2$ & $23 \pm 3$ & $(26 \pm 3)$ & $13 \pm 1$ & $17 \pm 2$ & $20 \pm 2$ & $(22 \pm 2)$ \\
\hline Kiruna $03 / 00$ & $15 \pm 1$ & $19 \pm 1$ & $22 \pm 1$ & $25 \pm 1$ & $13 \pm 1$ & $16 \pm 1$ & $18 \pm 1$ & $21 \pm 1$ \\
\hline Kiruna $03 / 03$ & $14 \pm 0$ & $17 \pm 0$ & $19 \pm 0$ & $21 \pm 0$ & $9 \pm 1$ & $13 \pm 1$ & $15 \pm 1$ & $18 \pm 1$ \\
\hline \multirow[t]{2}{*}{ EUPLEX 02/03 } & $15 \pm 0$ & $18 \pm 0$ & $(20 \pm 0)$ & & $12 \pm 0$ & $14 \pm 0$ & $(16 \pm 0)$ & \\
\hline & \multicolumn{4}{|c|}{$-{ }^{2} \varepsilon^{15} \mathrm{~N}_{\mathrm{app}}$} & \multicolumn{4}{|c|}{$-{ }^{1} \varepsilon^{15} \mathrm{~N}_{\mathrm{app}}$} \\
\hline $\ln \left(\mu / \mu_{\mathrm{T}}\right)$ & $>-0.6$ & -1.0 & -1.5 & -2.0 & $>-0.6$ & -1.0 & -1.5 & -2.0 \\
\hline India 03/87 & $27 \pm 2$ & & & & $11 \pm 1$ & & & \\
\hline India $04 / 99$ & $25 \pm 2$ & $(37 \pm 3)$ & & & $10 \pm 1$ & $(16 \pm 3)$ & & \\
\hline ASA 09/93 & $23 \pm 1$ & $33 \pm 2$ & $42 \pm 2$ & $(51 \pm 2)$ & $10 \pm 1$ & $13 \pm 1$ & $16 \pm 1$ & $(20 \pm 1)$ \\
\hline ASA $10 / 01$ & $27 \pm 1$ & $37 \pm 4$ & $(46 \pm 5)$ & & $9 \pm 1$ & $12 \pm 3$ & $(12 \pm 3)$ & \\
\hline ASA $10 / 02$ & $22 \pm 1$ & $33 \pm 9$ & $(40 \pm 12)$ & & $9 \pm 1$ & $16 \pm 3$ & $(23 \pm 4)$ & \\
\hline Gap 06/99 & $25 \pm 1$ & $31 \pm 1$ & $34 \pm 2$ & $38 \pm 2$ & $9 \pm 1$ & $10 \pm 1$ & $12 \pm 2$ & $14 \pm 2$ \\
\hline Kiruna $01 / 92$ & $23 \pm 1$ & $28 \pm 1$ & $32 \pm 1$ & $35 \pm 1$ & $11 \pm 0$ & $12 \pm 0$ & $13 \pm 0$ & $15 \pm 1$ \\
\hline Kiruna $02 / 92$ & $23 \pm 1$ & $31 \pm 2$ & $35 \pm 2$ & $39 \pm 3$ & $5 \pm 0$ & $12 \pm 3$ & $14 \pm 4$ & $17 \pm 5$ \\
\hline Kiruna 03/92 & $22 \pm 2$ & $26 \pm 1$ & $27 \pm 1$ & $28 \pm 1$ & $11 \pm 3$ & $8 \pm 2$ & $10 \pm 2$ & $11 \pm 2$ \\
\hline Kiruna 03/95 & $25 \pm 2$ & $30 \pm 3$ & $34 \pm 4$ & $(37 \pm 4)$ & $8 \pm 1$ & $10 \pm 2$ & $12 \pm 2$ & $(14 \pm 2)$ \\
\hline Kiruna $01 / 00$ & $22 \pm 2$ & $29 \pm 0$ & $32 \pm 0$ & $36 \pm 0$ & $4 \pm 1$ & $9 \pm 0$ & $12 \pm 0$ & $14 \pm 0$ \\
\hline Kiruna $03 / 03$ & $19 \pm 2$ & $24 \pm 2$ & $27 \pm 2$ & $29 \pm 2$ & $8 \pm 3$ & $9 \pm 2$ & $10 \pm 2$ & $12 \pm 2$ \\
\hline EUPLEX 02/03 & $22 \pm 0$ & $26 \pm 1$ & $(29 \pm 1)$ & & $9 \pm 0$ & $10 \pm 1$ & $(11 \pm 1)$ & \\
\hline
\end{tabular}

Further discrepancies to simple vertical reaction-diffusionadvection models arise at lower stratospheric levels. Specifically, average $\varepsilon^{15} \mathrm{~N}_{\text {app }}$ values at $\ln \left(\mu / \mu_{\mathrm{T}}\right)>-0.6$ are $(-18.5 \pm 0.9) \% o$ at tropical latitudes, $(-16.9 \pm 0.6) \% o$ at midlatitudes and $(-15.5 \pm 0.6) \%$ at polar latitudes. The pairwise differences are only significant at the $1 \sigma$ level, but all $\left|\varepsilon^{15} \mathrm{~N}_{\text {app }}\right|$ values are clearly less than half the absolute magnitude of the photolytic fractionation constant of about $-50 \%$, estimated for $\mathrm{N}_{2} \mathrm{O}$ broadband photolysis at a lower stratospheric temperature of $217 \mathrm{~K}$ (Kaiser et al., 2002b; von Hessberg et al. (2004) did not extend their ultraviolet cross section measurements to temperatures below $233 \mathrm{~K}$ ). The influence of spectral UV irradiance variations with altitude on the photolytic fractionation constant is small (Kaiser et al., 2003b). The discrepancy to the observations could be explained by at least a $25 \%$ contribution of photo-oxidation to the total $\mathrm{N}_{2} \mathrm{O}$ sink or by mixing effects other than vertical diffusion. Toyoda et al. (2004) based their analysis of the diminished apparent fractionation constants compared to their intrinsic values on photo-oxidation only, which led to estimated contributions of photo-oxidation between 70 and $80 \%$ to the total sink. However, this analysis is flawed because reductions of the intrinsic fractionation constant due to vertical diffusion and mixing were neglected. In Sect. 3.5, we show that correlations between isotope signature do indicate a contribution of photo-oxidation, but, for the most part, to a lesser degree than $70 \%$.

\subsubsection{Seasonal trends in $\varepsilon_{\text {app }}$}

Seasonal trends in the apparent fractionation constants are difficult to discern (Table 2), because the available balloon profiles are biased towards specific months, even if we combine our data-set with that of Toyoda et al. (2004). The two tropical profiles are both from spring, and the polar profiles are from winter/early spring. Only the mid-latitude profiles 


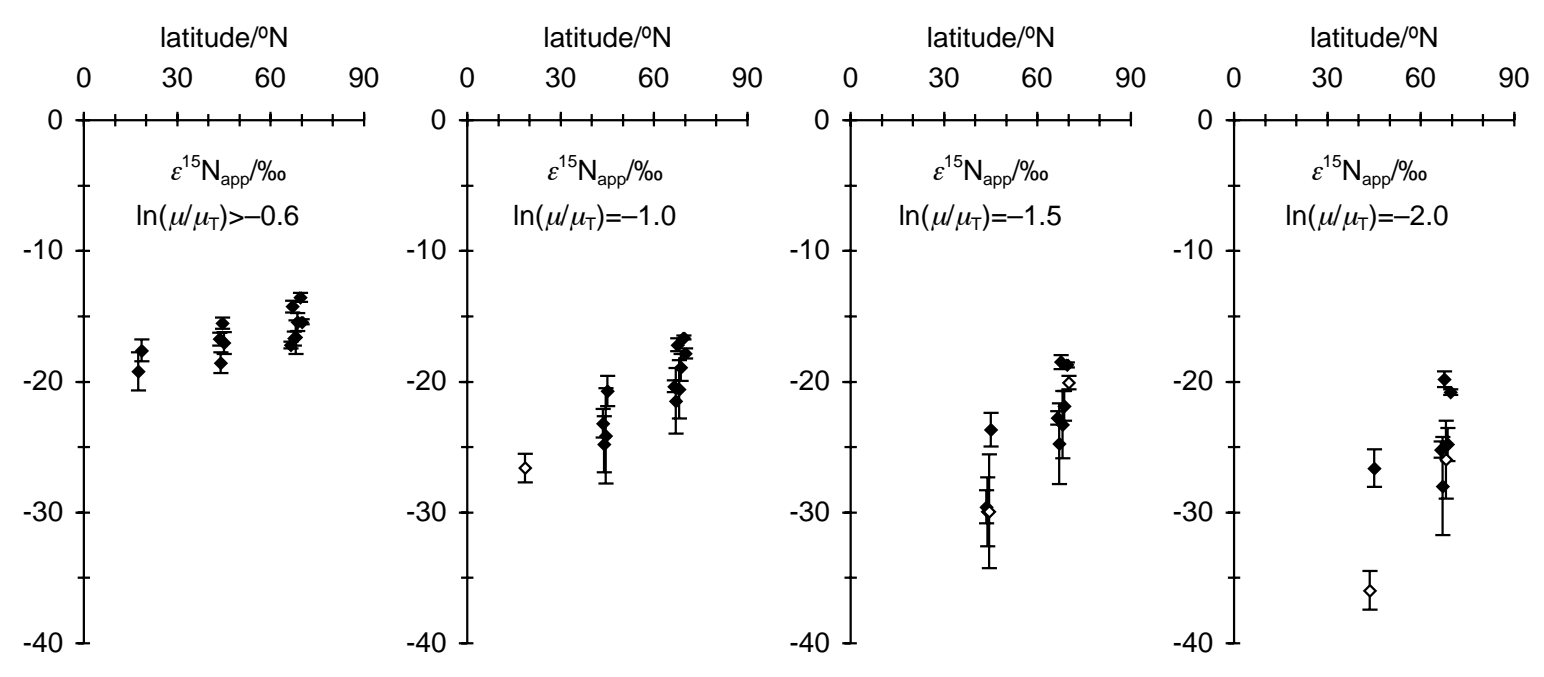

Fig. 7. Dependence of the apparent Rayleigh fractionation constant, $\varepsilon^{15} \mathrm{~N}_{\mathrm{app}}$, on latitude and remaining $\mathrm{N}_{2} \mathrm{O}$ fraction $\left(\mu / \mu_{\mathrm{T}}\right)$. For better visibility, data points at the same latitude have been separated by $0.5^{\circ}$ in the plot. Extrapolated values are shown as open symbols. The apparent fractionation constants have been derived from a linear fit to the data points with $\ln \left(\mu / \mu_{\mathrm{T}}\right)>-0.6$ and from the local slope of second-order polynomial fits at $\ln \left(\mu / \mu_{\mathrm{T}}\right)=-1.0,-1.5$, and -2.0 .

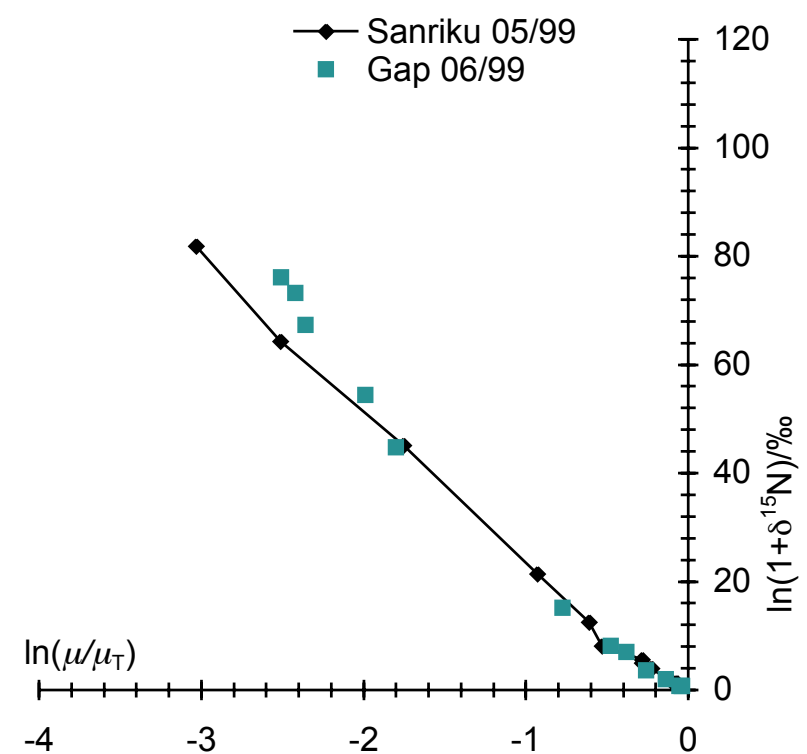

Fig. 8. Comparison between two stratospheric $\mathrm{N}_{2} \mathrm{O}$ profiles obtained at nearly the same time and same latitude, but at different longitudes (Gap 23 June 1999: 3-6 E, Sanriku 31 May 1999: $142^{\circ} \mathrm{E}$ ). The mixing ratios and $\delta$ values have been normalized to the same stratospheric entrance date. allow for a limited comparison between late spring/summer and autumn. The Gap 06/99 profile shows generally smaller $\left|\varepsilon_{\text {app }}\right|$ values than the fall ASA profiles. This is in contrast to model simulations by Morgan et al. (2004) that did not show variations in $\varepsilon_{\text {app }}$ at mid-latitudes. However, the simulations did show variations at polar and tropical latitudes, which were attributed to transport effects. Therefore, we tentatively associate the observed variations at mid-latitudes with transport effects as well.

Similar variations were observed by Toyoda et al. (2004) for balloon profiles acquired over Japan. For example, in a plot of isotope versus mixing ratios (not shown), the Sanriku 08/00 and 09/98 profiles behave similarly to the ASA 09/93, 10/01 and 09/02 samples (late summer/fall), whereas the Sanriku 05/99, 05/01 and 06/90 profiles bear closer resemblance to the Gap 06/99 profile and the polar samples (spring/early summer). Modeling attempts therefore have to consider seasonal and meridional variations of the $\mathrm{N}_{2} \mathrm{O}$ isotope signature if quantitative agreement with observations is to be achieved. However, it might even be necessary to include longitudinal variations as a comparison between the Gap 06/99 and Sanriku 05/99 profiles shows (Fig. 8). Both profiles were obtained at nearly the same time of the year (three weeks apart) and nearly the same latitude $\left(44^{\circ} \mathrm{N}\right.$ and $39^{\circ} \mathrm{N}$ ), but nevertheless show distinct differences especially in the middle stratosphere. 


\subsection{Influence of mixing on $\mathrm{N}_{2} \mathrm{O}$ isotope ratios}

In our data interpretation so far, we have focused on vertical diffusive effects on the apparent fractionation constants. However, meridional transport and mixing can also influence the observed relationship between $\mathrm{N}_{2} \mathrm{O}$ isotope signatures and mixing ratios (Kaiser et al., 2002a). Specifically, Park et al. (2004) mentioned two mixing scenarios, "end-member mixing" and "continuous weak mixing" (Plumb et al., 2000), in the context of $\delta-\mu$ relationships for the polar vortex. In the following, we will investigate to what degree these scenarios can explain the observed stratospheric $\mathrm{N}_{2} \mathrm{O}$ isotope profiles, not only in the polar vortex, but also in other stratospheric regions. We start with "end-member mixing", followed by "continuous weak mixing", with a short mathematical introduction to each scheme.

\subsubsection{Theory}

"End-member mixing" corresponds to the mixing of air masses in different relative volumetric ratios. It is "linear" in terms of concentrations; however, $\delta$ values have to be weighted by the corresponding concentrations. For two air masses, A and B, this can be expressed mathematically by the following relationships for two air masses, A and B (Kaiser et al., 2002a):

$c=x_{\mathrm{A}} c_{\mathrm{A}}+\left(1-x_{\mathrm{A}}\right) c_{\mathrm{B}}$

$c \delta=x_{\mathrm{A}} c_{\mathrm{A}} \delta_{\mathrm{A}}+\left(1-x_{\mathrm{A}}\right) c_{\mathrm{B}} \delta_{\mathrm{B}}$

The symbol $x$ designates a volumetric air mass fraction, the symbol $c$ designates concentrations. Indices denote the air mass. Non-indexed symbols correspond to the mixed air mass. If both air masses have the same density, mixing ratios $(\mu)$ can be used instead of concentrations. This condition is assumed to be always valid, because both air masses have to be at the same altitude in order to mix. Therefore, we replace concentrations by mixing ratios and solve Eq. (2) for $x$ :

$x=\frac{\mu_{\mathrm{B}}-\mu}{\mu_{\mathrm{B}}-\mu_{\mathrm{A}}}$,

which is then substituted into Eq. (3) and solved for $\delta$ :

$$
\begin{aligned}
\delta & =\frac{\mu_{\mathrm{B}}-\mu}{\mu_{\mathrm{B}}-\mu_{\mathrm{A}}} \frac{\mu_{\mathrm{A}} \delta_{\mathrm{A}}}{\mu}+\frac{\mu-\mu_{\mathrm{A}}}{\mu_{\mathrm{B}}-\mu_{\mathrm{A}}} \frac{\mu_{\mathrm{B}} \delta_{\mathrm{B}}}{\mu} \\
& =\frac{\mu_{\mathrm{B}} \delta_{\mathrm{B}}-\mu_{\mathrm{A}} \delta_{\mathrm{A}}}{\mu_{\mathrm{B}}-\mu_{\mathrm{A}}}+\frac{\delta_{\mathrm{B}}-\delta_{\mathrm{A}}}{\mu_{\mathrm{B}}^{-1}-\mu_{\mathrm{A}}^{-1}} \frac{1}{\mu}
\end{aligned}
$$

Thus, two-end-member mixing should lead to linear relationships between $\delta$ values and inverse mixing ratios $\left(\mu^{-1}\right)$. The graphical presentation of this kind of relationship is often attributed to a paper by Keeling (1958) and colloquially referred to as "Keeling plot", even though the original paper only mentions the mathematical relationship, but does not show a plot of $\delta$ values against inverse mixing ratios. A Keeling plot is better suited to diagnose mixing than $\delta-\mu$ plots
(Park et al., 2004), because both mixing and Rayleigh fractionation appear as similar non-linear curves in a $\delta-\mu$ plot. Note that after correction for a sign error, Eq. (8) derived for oceanic $\mathrm{O}_{2}$ in Bender (1990) corresponds to Eq. (5) above.

Note that the analysis of mixing using Keeling plots is generally restricted to two end-members only. These two endmembers could correspond to different pairs of air masses, for example, stratospheric polar vortex and mid-latitude extra-vortex air, lower stratospheric and upper tropospheric air, or upper stratospheric/mesospheric air and middle stratospheric air. This is of course an idealistic representation of real atmospheric transport processes, and the purpose of the present analysis can only be to investigate which features of the stratospheric $\mathrm{N}_{2} \mathrm{O}$ isotope distribution can be described by simple two-end-member mixing.

\subsubsection{Evidence for mixing from balloon samples}

Without restriction of generality, we develop our analysis using $\delta^{15} \mathrm{~N}$ (the other isotope signatures behave very similarly, see Sect. 3.5). The $\delta^{15} \mathrm{~N}$ values of the present stratospheric dataset have been plotted against their inverse mixing ratios in Fig. 9. Most of the samples cluster near the origin of the plot. At this scale, the tropical, mid-latitude ASA, and low-altitude polar samples follow a linear relationship. However, the mid-latitude Gap 06/99 and the high-altitude polar samples deviate from this linear relationship at mixing ratios below $180 \mathrm{nmol} \mathrm{mol}^{-1}$. Contrary to the suggestion of Park et al. (2004), the behavior of the latter samples cannot be explained by simple mixing between two well-defined end-members, because they do not describe a linear array in $\delta-\mu^{-1}$ space.

Figure 10 shows the same data as in Fig. 9 restricted to mixing ratios above $50 \mathrm{nmol} \mathrm{mol}^{-1}$. Again, the non-linearity of the $\delta$ vs. $\mu^{-1}$ relationship for polar samples is apparent, while the mid-latitude ASA samples closely follow a linear relationship down to mixing ratios of $60 \mathrm{nmol} \mathrm{mol}^{-1}$. The green line in Fig. 10 shows a linear fit to the ASA 09/93 data, $\delta^{15} \mathrm{~N}($ ASA $09 / 93)=4.0 \cdot 10^{-9} \mu^{-1}-12.4 \%$ o $\left(R^{2}=0.997\right)$. This indicates that the mid-latitude $\mathrm{N}_{2} \mathrm{O}$ isotope profiles could be described by a simple two-end-member mixing relationship, to a good degree of approximation. The advantage of using the relationship between $\delta$ and the inverse mixing ratio to diagnose mixing is that it is based on a single trace gas only. If transport is fast with respect to photochemistry of $\mathrm{N}_{2} \mathrm{O}$, then linear mixing relationships between $\delta$ and $\mu^{-1}$ result. In contrast, $\mathrm{CH}_{4}-\mathrm{N}_{2} \mathrm{O}$ correlations are only linear if transport is fast with respect to the chemistry of both trace gases. This may explain why the mid-latitude samples follow a two-endmember mixing relationship closely in $\delta-\mu^{-1}$ space, but not in $\mathrm{CH}_{4}-\mathrm{N}_{2} \mathrm{O}$ space (Fig. 4). Nevertheless, the linearity of the $\delta-\mu^{-1}$ relationship is not perfect and the scatter about the linear regression line is significantly larger than the analytical errors. It must therefore be due to natural variability. Moreover, even the mid-latitude ASA samples show a 

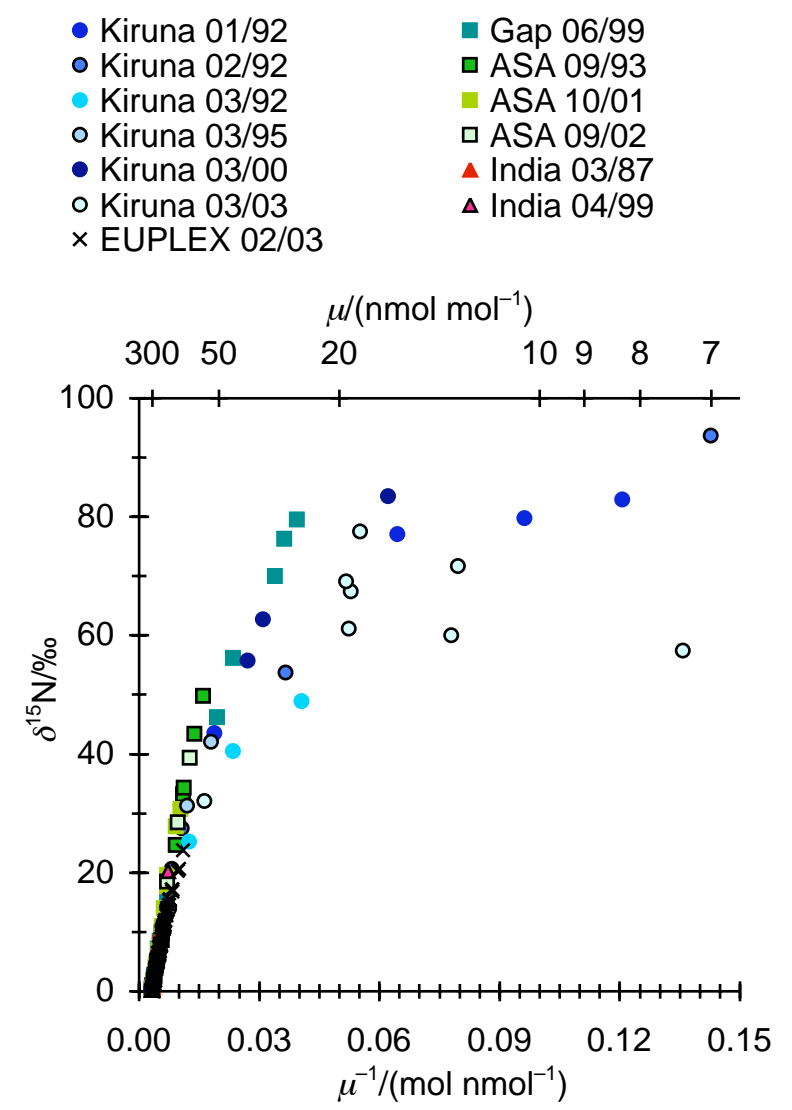

Fig. 9. Mixing plot of the bulk nitrogen isotope ratio. Two-endmember mixing would lead to a linear relationship in a $\delta-\mu^{-1}$ plot. One Kiruna $03 / 03$ sample at $0.283 \mathrm{~mol} \mathrm{nmol}^{-1} / 139.6 \%$ o $\left(3.5 \mathrm{nmol} \mathrm{mol}^{-1}\right)$ has been omitted in order to show details for samples with higher mixing ratios more clearly.

decrease in the slope towards lower mixing ratios, which indicates that - despite the strong correlation between $\delta$ and $\mu^{-1}$ - it cannot be two-end-member mixing alone that is responsible for the mid-latitude $\mathrm{N}_{2} \mathrm{O}$ isotope profile.

\subsubsection{Evidence for mixing from aircraft samples}

Figure 10 also shows two dense data-sets from aircraft campaigns into and out of the Arctic polar vortex (SOLVE 2000; see Park et al., 2004; and EUPLEX 2003). Again, for mixing ratios above about $200 \mathrm{nmol} \mathrm{mol}^{-1}, \delta$ follows a linear relationship relative to $\mu^{-1}$. However, overall the relationship is curved. E.g., for EUPLEX 2003, the $\delta^{15} \mathrm{~N}$ values can be approximated by a second-order polynomial: $\quad \delta^{15} \mathrm{~N}\left(\right.$ EUPLEX 2003) $=-1.4 \cdot 10^{-16} \mu^{-2}+4.9 \cdot 10^{-9}$ $\mu^{-1}-14.2 \%$ o $\left(R^{2}=0.998\right)$. Neither linear mixing nor Rayleigh fractionation can explain the observed behavior at mixing ratios below $200 \mathrm{nmol} \mathrm{mol}^{-1}$. A different transport scheme must therefore be invoked, possibly "continuous weak mixing" (Sect. 3.4).

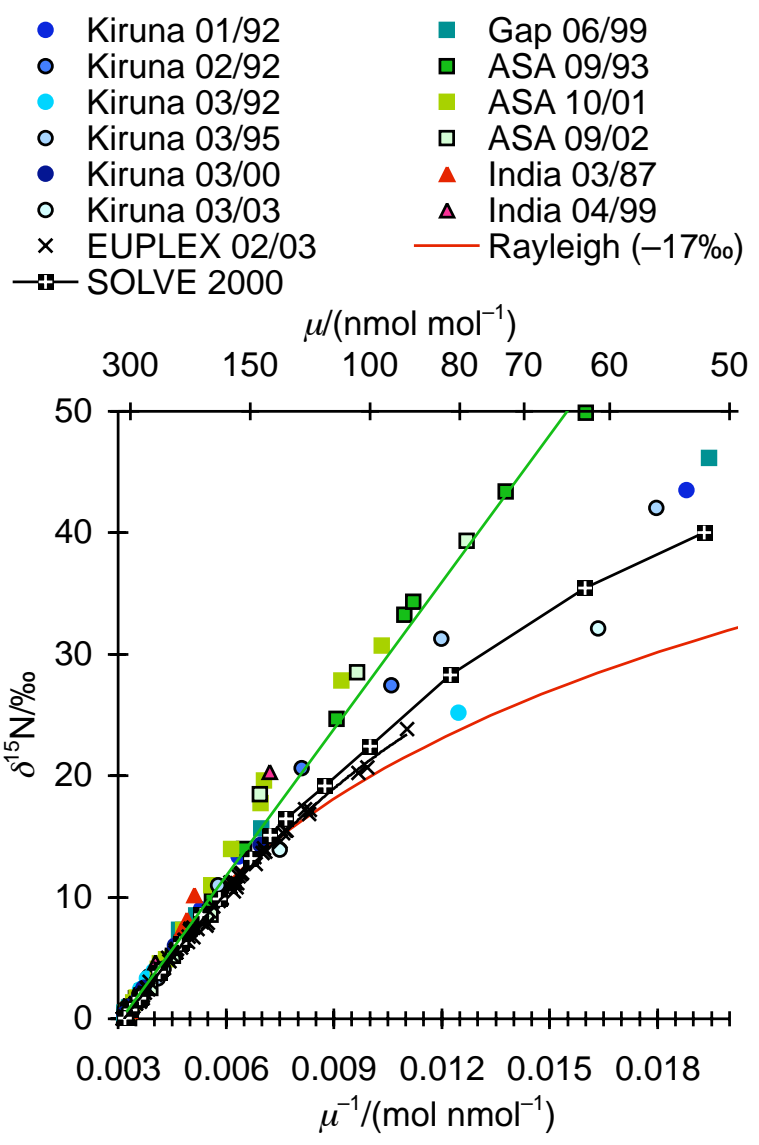

Fig. 10. Linear two-end-member mixing for some midlatitude samples, e.g. $\delta^{15} \mathrm{~N}($ ASA $09 / 93)=4.0 \cdot 10^{-9} \mu^{-1}-12.4 \%$ o $\left(R^{2}=0.997\right)$; weak mixing (?) at polar latitudes, $\delta^{15} \mathrm{~N}$ (EUPLEX $2003)=-1.4 \cdot 10^{-16} \mu^{-2}+4.9 \cdot 10^{-9} \mu^{-1}-14.2 \%$ o $\left(R^{2}=0.998\right)$. Also shown are a Rayleigh-fractionation curve with a fractionation constant of $\varepsilon=-17 \%$ and the results of the SOLVE 2000 aircraft campaign (Park et al., 2004).

Figure 11 demonstrates this behavior in greater detail. The left panel shows the residual for the EUPLEX 2003 and SOLVE 2000 data after a linear least-squares fit of $\delta^{15} \mathrm{~N}$ vs. $\mu^{-1}$ has been applied to the data between 200 and $320 \mathrm{nmol} \mathrm{mol}^{-1}$. The right panel shows an analogous fit of the data between 170 and $320 \mathrm{nmol} \mathrm{mol}^{-1}$. In both cases, the residuals become very large below the lower boundary of the fit region. However, in the $170 \mathrm{nmol} \mathrm{mol}^{-1}$ case, the residuals also show a pattern in the range between 170 and $320 \mathrm{nmol} \mathrm{mol}^{-1}$, with foremost positive residuals in the 170 to $260 \mathrm{nmol} \mathrm{mol}^{-1}$ range and negative residuals in the 260 to $320 \mathrm{nmol} \mathrm{mol}^{-1}$ range. Such a pattern is absent in the 200 to $320 \mathrm{nmol} \mathrm{mol}^{-1}$ range. This neatly demonstrates the suitability of linear mixing to describe the lower polar vortex relationship between $\mathrm{N}_{2} \mathrm{O}$ mixing ratios and isotope ratios, for mixing ratios above approximately $200 \mathrm{nmol} \mathrm{mol}^{-1} \cdot R^{2}$ values are less indicative of the suitability of the fit than a possible pattern in the residuals. For completeness, we note 

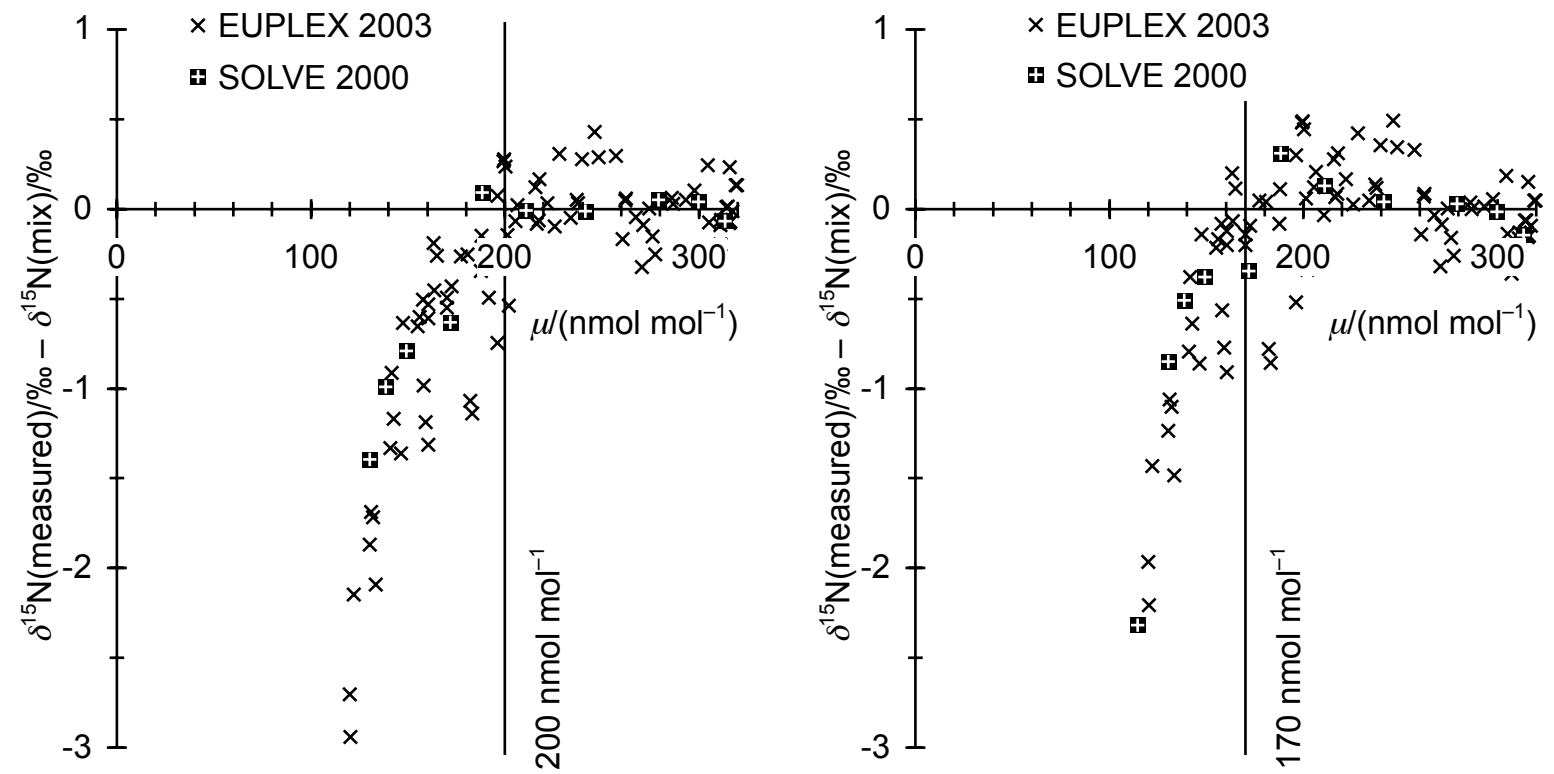

Fig. 11. Residuals of linear fits to polar aircraft $\mathrm{N}_{2} \mathrm{O}$ measurements. No structure is visible in the residuals for a lower cut-off of $200 \mathrm{nmol} \mathrm{mol}^{-1}$ (left panel), but if a lower cut-off of $170 \mathrm{nmol} \mathrm{mol}^{-1}$ (right panel) is used for the linear fits, samples closer to $170 \mathrm{nmol} \mathrm{mol}^{-1}$ fall mostly above the $\mathrm{x}$-axis, while samples closer to $300 \mathrm{nmol} \mathrm{mol}^{-1}$ fall mostly below the x-axis.

that $R^{2}$ improves from 0.997 to 1.000 (SOLVE) and from 0.993 to 0.994 (EUPLEX) upon change of the lower boundary from $170 \mathrm{nmol} \mathrm{mol}^{-1}$ to $200 \mathrm{nmol} \mathrm{mol}^{-1}$.

As noted above, a Rayleigh plot yields a linear array in $\ln (1+\delta)-\ln \left(\mu / \mu_{\mathrm{T}}\right)$ space, for $\ln \left(\mu / \mu_{\mathrm{T}}\right)>-0.6$, corresponding to mixing ratios $>160 \mathrm{nmol} / \mathrm{mol}$, so that the compact relationship of $\delta$ and $\mu$ for mixing ratios $>200 \mathrm{nmol} \mathrm{mol}^{-1}$ could potentially be explained by Rayleigh fractionation under the influence of vertical advection and diffusion. However, the slope of a linear fit to the Rayleigh plot is smaller even than the effective fractionation constant under transport-limited conditions, i.e. about half the value of the intrinsic fractionation constant, and this simple explanation model alone is therefore not sufficient.

The failure of "two-end-member mixing" to describe the $\mathrm{N}_{2} \mathrm{O}$ isotope variations in polar aircraft samples with $\mathrm{N}_{2} \mathrm{O}$ mixing ratios below about $200 \mathrm{nmol} \mathrm{mol}^{-1}$ can be interpreted as a hint to the mechanism by which upper stratospheric air in the polar vortex mixes with extra-vortex mid-latitude air. Rather than a single, "late" mixing event, "continuous weak mixing" between vortex and extra-vortex air might be the relevant mechanism. This process was first proposed and developed conceptually by Plumb et al. (2000). In Sect. 3.4, we include isotopes in a two-dimensional transport scheme similar to the one used by Plumb et al. and evaluate whether the observed isotope variations could indeed be due to continuous weak mixing.

\subsubsection{Upper stratospheric or mesospheric air intrusions}

Figures 9 and 10 also show that polar balloon samples with $\mathrm{N}_{2} \mathrm{O}$ mixing ratios below $100 \mathrm{nmol} \mathrm{mol}^{-1}$ strongly deviate from the mid-latitude $\delta-\mu^{-1}$ relationship, especially in the case of the Kiruna $03 / 03$ profile. The latter profile comprises three samples with mixing ratios between 7 and $19 \mathrm{nmol} \mathrm{mol}^{-1}$, but nearly identical $\delta^{15} \mathrm{~N}$ values around $60 \%$. They were obtained at altitudes of $23.9 \mathrm{~km}$ $\left(7 \mathrm{nmol} \mathrm{mol}^{-1}\right), \quad 25.3 \mathrm{~km}\left(13 \mathrm{nmol} \mathrm{mol}^{-1}\right)$, and $28.4 \mathrm{~km}$ $\left(19 \mathrm{nmol} \mathrm{mol}^{-1}\right)$. The nearly horizontal array of these three samples in $\delta-\mu^{-1}$ space can be explained by mixing with $\mathrm{N}_{2} \mathrm{O}$-free upper stratospheric or mesospheric air, descending diabatically in the polar vortex, as was first suggested by Toyoda et al. (2004). In the polar night, no photochemical destruction of $\mathrm{N}_{2} \mathrm{O}$ occurs and transport is therefore the only process that can influence the relationship between $\mathrm{N}_{2} \mathrm{O}$ isotope and mixing ratios. The fact that the high-latitude Kiruna 03/03 samples contained mesospheric air agrees with $\mathrm{CO}_{2}$ and $\mathrm{SF}_{6}$ analyses of the same set of samples analyzed here (Engel et al., 2006) and concomitant aerosol measurements (Curtius et al., 2006). Meteorological observations and age of air estimates derived from $\mathrm{CO}_{2}$ and $\mathrm{SF}_{6}$ measurements showed that in the boreal winter of 2002/2003, there was indeed an mesospheric intrusion into the Arctic polar vortex, which descended in the vortex throughout the winter. At the time of the late-winter Kiruna 03/03 balloon profile, the polar vortex had already started to break up. This is in line with increasing $\mathrm{N}_{2} \mathrm{O}$ mixing ratios at altitudes above $23 \mathrm{~km}$, which indicate that the essentially $\mathrm{N}_{2} \mathrm{O}$-free upper stratospheric or 
mesospheric air had started to mix with stratospheric extravortex air. Based on Fig. 9, a similar event may have occurred in the winter of 1992 , because also then, similar $\delta^{15} \mathrm{~N}$ were observed over a large range of mixing ratios in the middle stratosphere.

\subsection{Influence of continuous weak mixing on $\mathrm{N}_{2} \mathrm{O}$ isotope ratios}

Plumb et al. (2000) showed that tracer-tracer relationships in the Arctic vortex are better interpreted by continuous weak mixing across the vortex edge than by simple two-endmember mixing. They used a conceptual two-dimensional model to illustrate the effects of continuous mixing and showed that compact tracer-tracer relationships develop for exterior air and vortex air, with a small transition region in between. We have used the same conceptual model as Plumb et al. (2000) to investigate whether continuous weak mixing is a useful concept to better interpret the isotope variations in our polar vortex samples.

Briefly, a simple advective-diffusive model in cylindrical geometry is set up with 41 grid points in the $r$-direction and 101 grid points in the z-direction, corresponding to latitude and altitude. Latitudinal downwelling with vertical velocity $w(r)=-\cos \left(\frac{1}{2} \pi r\right)$ is assumed, so that the maximum downwelling occurs at the pole (note the correction of a typo in the definition of $w$ in Plumb et al., 2000). The model is dimensionless with spatial co-ordinates from 0 to 1 and mixing ratios expressed relative to their value at the tropopause $\left(\chi=\mu / \mu_{\mathrm{T}}\right)$. The unit of time is the time it takes for air to descend at the pole from top to bottom of the domain. With $K$ being the horizontal (eddy) diffusivity, the normalized mixing ratios evolve according to

$$
\frac{\partial \chi}{\partial t}=-w \frac{\partial \chi}{\partial z}+\frac{1}{r} \frac{\partial}{\partial r}\left(r K \frac{\partial \chi}{\partial r}\right) .
$$

The horizontal diffusivity $K$ was defined by Plumb et al. (2000) as piecewise uniform over several regions (numerical values correspond to the first case discussed in Plumb et al., 2000):

\begin{tabular}{lll} 
Vortex & $0.2<z \leq 1.0$ & $0.0 \leq r<0.3$ \\
& $K=K_{v}=0.25$ & \\
Edge region & $0.2<z \leq 1.0$ & $0.3<r<0.4$ \\
& $K=K_{e}=0.05$ & \\
Exterior & $0.0 \leq z \leq 1.0$ & $0.4<r \leq 1.0$ \\
& $K=K_{m}=2.0$ \\
Subvortex & $0.1<z<0.2$ & $0.0 \leq r<0.3$ \\
& $K:$ linear transition between $K_{m}$ and $K_{v}$ \\
& \multicolumn{2}{c}{} \\
Subvortex & $0.1<z<0.2$ & $0.3<r<0.4$ \\
& $K:$ linear transition between $K_{m}$ and $K_{e}$
\end{tabular}

$$
\begin{gathered}
\text { Subvortex } 0.0 \leq z<0.1 \quad 0.0 \leq r<0.4 \\
K=K_{m}
\end{gathered}
$$

We use continuously defined horizontal diffusivities instead with the same transition points at $r=0.3, r=0.4$ and $z=0.1$ as in Plumb et al. (2000):

$$
\begin{aligned}
& K_{r}(r)=\left(K_{v}-K_{e}\right) e^{-(r+0.7)^{38}}+\left(K_{m}-K_{e}\right) e^{-(r-1.4)^{62}}+K_{e} \\
& K_{r}(r, z)=\left(K_{r}-K_{m}\right) e^{-(z-1.1)^{38}}+K_{m}
\end{aligned}
$$

As boundary conditions at the exterior edge of the domain $(r=1)$, we set $\chi(1, z)=\frac{1}{2}\left[\cos \left(\pi z^{2}\right)+1\right]$, at the bottom $\chi(r, 0)=1$ and at the top $\chi(r, 1)=0$. At $r=0$, we specify $\partial \chi / \partial r=0$. For the sake of argument, we assume that the mixing ratio of the minor isotope at the exterior edge is given by $\chi^{\prime}(1, z)=\chi(1, z)^{1+\varepsilon}$ with $\varepsilon=-38 \%$ (corresponding to the ${ }^{15} \mathrm{~N}$ isotope effect used in Figs. 5 and 6).

The resulting steady-state dependence of the isotope ratio on $\chi=\mu / \mu_{\mathrm{T}}$ is shown in Fig. 12a. Two compact $\delta-\mu / \mu_{\mathrm{T}}$ relationships of finite width develop in the vortex and in the exterior, just as found by Plumb et al. (2000) for conceptual tracer-tracer relationships between $\mathrm{NO}_{\mathrm{y}}-\mathrm{N}_{2} \mathrm{O}$ and $\mathrm{CFCl}_{3}-$ $\mathrm{N}_{2} \mathrm{O}$. A horizontal (quasi-isentropic) cross-section at $z=0.7$ clearly shows a different $\delta-\mu / \mu_{\mathrm{T}}$ relationship than expected for two-end-member mixing. The modeled tracer-tracer relationships can also be evaluated in a Rayleigh plot (Fig. 12c) or as mixing plot (Fig. 12e). The Rayleigh plot (Fig. 12c) for a constant value of $r$ bears some similarity to a Rayleigh fractionation plot for a one-dimensional reaction-diffusionadvection scheme with an isotope effect below the intrinsic isotope effect although in the present case no chemistry takes place in the model region. The mixing plot (Fig. 12e) shows that continuous weak mixing leads to curved quasi-isentropic arrays in a $\delta-\mu^{-1}$ plot, which does suggest that the polar $\mathrm{N}_{2} \mathrm{O}$ isotope profiles are more likely caused by continuous weak mixing than by two-end-member mixing.

A comparison of the continuous weak mixing model with stratospheric measurements (Figs. 12b, d, and f) shows that it captures the features in the stratospheric $\mathrm{N}_{2} \mathrm{O}$ isotope data for mixing ratios above $200 \mathrm{nmol} \mathrm{mol}^{-1}$, just as the other two conceptual models discussed here (modified Rayleigh fractionation in a one-dimensional reaction-diffusion-advection scheme and two-end-member mixing). However, only the continuous weak mixing model seems to simulate the qualitative behavior at both high and low mixing ratios. It needs to be emphasized, though, that any form of quantitative agreement between measurements and the continuous weak mixing model used here is purely coincidental, since the model parameters have been chosen arbitrarily. We are therefore just comparing general, qualitative features.

Modified Rayleigh fractionation leads to linear correlations between $\ln (1+\delta)$ and $\ln \left(\mu / \mu_{\mathrm{T}}\right)$. It thus fails to simulate the curvature of the EUPLEX measurements. Two-end-member mixing should yield linear correlations 

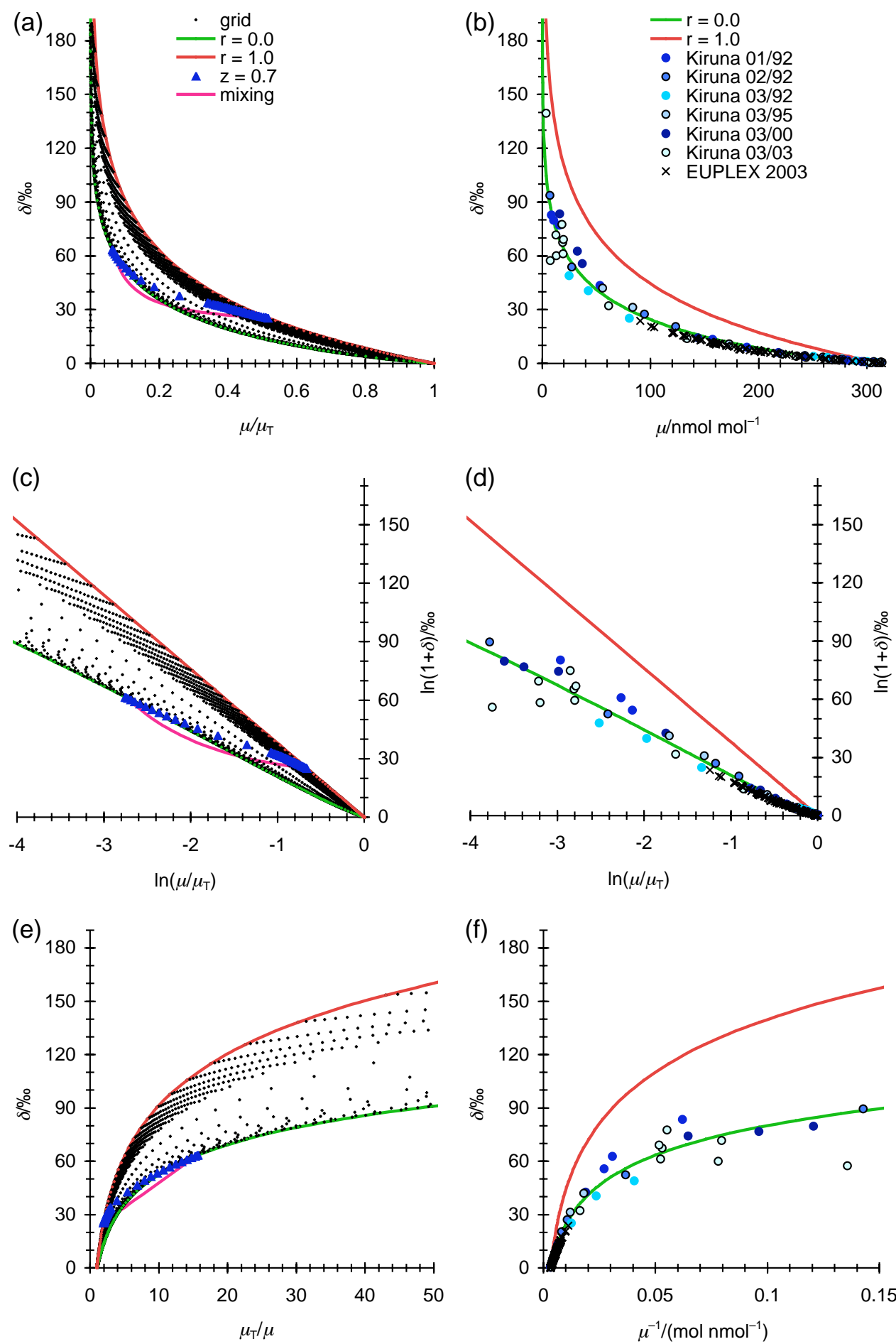

Fig. 12. (a) Plot of $\delta$ value vs. normalized mixing ratio for a continuous weak mixing model on a $41 \times 101$ ( $r \times z)$ grid (based on Plumb et al., 2000). Grid boundaries at $r=0.0$ and $r=1.0$, a horizontal cross-section at $z=0.7$ as well as a hypothetical mixing curve between the ( 0.0 , $0.7)$ and $(1.0,0.7)$ end-members are indicated. Continuous weak mixing leads to a clustering of the $\delta$ values near the curves defined by the grid boundaries. These correspond to the intravortex (polar) and extravortex (midlatitude) regions. The values the $r=1.0$ grid boundary corresponds to the assumed constant isotope effect of $\varepsilon=-38 \%$. (b) Comparison between polar stratospheric measurements from balloon and aircraft campaigns at Kiruna with continuous weak mixing model results at the grid boundaries. The normalized mixing ratios from the model runs have been multiplied by an $\mathrm{N}_{2} \mathrm{O}$ mixing ratio of $314 \mathrm{nmol} \mathrm{mol}^{-1}$ (1998) to facilitate the comparison to stratospheric measurements. (c, d) Same as (a, b), but now plotted as $\ln (1+\delta)$ vs. $\ln \left(\mu / \mu_{\mathrm{T}}\right)$. (e, f) Same as (a, b), but now plotted as $\delta$ value vs. normalized inverse mixing ratio, $\mu_{\mathrm{T}} / \mu$, or inverse mixing ratio, $\mu^{-1}$. 
between $\delta$ and $\mu^{-1}$, and thus fails at mixing ratios below $200 \mathrm{nmol} \mathrm{mol}^{-1}$. In contrast, in the continuous weak mixing model, an increase of the magnitude of the apparent fractionation constant with decreasing $\mathrm{N}_{2} \mathrm{O}$ mixing ratio between is noticeable between $\ln \left(\mu / \mu_{\mathrm{T}}\right)=0$ and $\ln \left(\mu / \mu_{\mathrm{T}}\right)=-0.6$, which means that this conceptual transport model comes closer to explaining the observed stratospheric $\mathrm{N}_{2} \mathrm{O}$ isotope variations. This does not mean that the conceptual model presented here should be construed as an attempt to realistically simulate stratospheric transport and chemistry. It only serves to illustrate that the level of precision we have achieved in stratospheric $\mathrm{N}_{2} \mathrm{O}$ isotope measurements warrants moving beyond simple Rayleigh fractionation and two-end-member mixing models. For a full appraisal of the observations two- or three-dimensional atmospheric chemistry models are needed and are in fact already being used (McLinden et al., 2003; Morgan et al., 2004).

\subsection{Correlations between position-dependent isotope en- richments}

In this final section, we demonstrate that position-dependent $\mathrm{N}_{2} \mathrm{O}$ isotope measurements are not only useful to make inferences about stratospheric transport, but also bear some signal of variations in the relative contributions of the two photochemical $\mathrm{N}_{2} \mathrm{O}$ sinks in the stratosphere, photolysis and reaction with $\mathrm{O}\left({ }^{1} \mathrm{D}\right)$, as first suggested by Röckmann et al. (2001) and Toyoda et al. (2001) and further elaborated by Kaiser (2002) and Kaiser et al. (2002a, b, 2003b). The effects of transport and mixing on the correlation between positiondependent isotope enrichments essentially cancel (Kaiser et al., 2002a). This can be exploited to discern variations in stratospheric chemistry with respect to altitude or latitude. The strongest effects on the correlation between positiondependent isotope enrichment are expected from the varying contribution of $\mathrm{N}_{2} \mathrm{O}+\mathrm{O}\left({ }^{1} \mathrm{D}\right)$ reaction to the total $\mathrm{N}_{2} \mathrm{O}$ sink, with smaller influences from changes in temperature and the ultraviolet spectrum (Kaiser, 2000; Kaiser et al., 2002a, b, 2003b). Based on their data-set of polar aircraft samples, Park et al. (2004) questioned the possibility to detect altitude variations in the relative contribution of the photolysis and $\mathrm{O}\left({ }^{1} \mathrm{D}\right)$ sink, but proposed instead that meridional differences in the correlation between different isotope signatures might be apparent, with a stronger influence of the $O\left({ }^{1} D\right)$ sink at tropical latitudes.

Toyoda et al. (2004) calculated the contribution of the $\mathrm{O}\left({ }^{1} \mathrm{D}\right)$ sink from apparent stratospheric fractionation constants and the fractionation constants measured for UV photolysis and the reaction of $\mathrm{N}_{2} \mathrm{O}$ with $\mathrm{O}\left({ }^{1} \mathrm{D}\right)$ in the laboratory. However, they did not allow for transport effects and the ensuing reduction of the apparent fractionation constant from its intrinsic (i.e., photochemical) value.

Specifically, Toyoda et al. (2004) used the following equation to compute the overall fractionation in the stratospheric
$\mathrm{N}_{2} \mathrm{O}$ sink reactions

$\varepsilon_{\text {sink }}=x \varepsilon_{\text {photo }}+(1-x) \varepsilon_{\text {oxy }}$

where $\varepsilon_{\text {photo }}$ and $\varepsilon_{\text {oxy }}$ are the isotopic fractionation by photolysis and photo-oxidation, respectively, and $x$ is the share of photolysis in the total $\mathrm{N}_{2} \mathrm{O}$ sink. This equation in itself is correct, but the authors then proceed to equate $\varepsilon_{\text {sink }}$ with $\varepsilon_{\text {app }}$ and compute $x$ in the lower stratosphere from Eq. (6). This led them to conclude that the contribution of photolysis to the total $\mathrm{N}_{2} \mathrm{O}$ sink is significantly less than the value of 90\% derived from integrating stratospheric chemistry models (Minschwaner et al., 1993). However, this calculation does not take into account that transport effects prevent the full intrinsic photochemical isotope fractionation from being expressed in the stratosphere, as Toyoda et al. (2004) acknowledge in the subsequent section of their paper. In a simple one-dimensional reaction-diffusion scheme, the effective fractionation constant can be calculated from the intrinsic fractionation constant and the reaction and transport time-scales as follows (Kaye, 1987; Kaiser, 2002; Kaiser et al., 2002a; Brenninkmeijer et al., 2003):

$\varepsilon_{\mathrm{eff}}=\frac{\sqrt{1+Q\left(1+\varepsilon_{\text {sink }}\right)}-1}{\sqrt{1+Q}-1}-1$

$Q$ is the ratio of transport time-scale, $\tau_{\text {trans }}=4 H_{\mathrm{av}}^{2} / K_{z}$, and reaction time-scale, $\tau_{\text {chem }}=1 / k$. Kaiser (2002) and Brenninkmeijer et al. (2003) have shown that $\varepsilon_{\text {app }}$ varies between $\sqrt{1+\varepsilon_{\text {sink }}}-1 \approx \frac{1}{2} \varepsilon_{\text {sink }}$ and $\varepsilon_{\text {sink }}$. Following Morgan et al. (2004), the following approximation of Eq. (7) can be made for $\varepsilon_{\text {sink }} \rightarrow 0$

$\varepsilon_{\mathrm{eff}} \approx \frac{1}{2} \varepsilon_{\text {sink }}\left(1+\frac{1}{\sqrt{1+Q}}\right)$,

which illustrates that, within the limits of the approximation made, isotope effects and transport effects can be separated.

In order to apply Eq. (7) to compute $x$, it is necessary to know the value of $Q$, a quantity which is not generally known. It is therefore useful to look at ratios of different intramolecular fractionation constants, or, more directly, at correlations between different intramolecular $\mathrm{N}_{2} \mathrm{O}$ isotope signatures.

Toyoda et al. (2004) indicated that their dataset was not comprehensive enough to use correlations between individual $\mathrm{N}_{2} \mathrm{O}$ isotope signatures to derive the contribution of different photochemical $\mathrm{N}_{2} \mathrm{O}$ sinks.

With the present comprehensive, high-precision dataset, we are now revisiting the suggested variations of $\eta_{\mathrm{app}}$, i.e., the correlation between $\ln \left(1+{ }^{2} \delta^{15} \mathrm{~N}\right)$ and $\ln \left(1+{ }^{1} \delta^{15} \mathrm{~N}\right)$, and $\psi_{\text {app }}$, i.e., the correlation between $\ln \left(1+\delta^{15} \mathrm{~N}\right)$ and $\ln \left(1+\delta^{18} \mathrm{O}\right)$ (Kaiser, 2002; Kaiser et al., 2002a; Park et al., 2004). Instead of taking the ratio of apparent fractionation constants over larger stratospheric regions (Röckmann et al., 2001; Toyoda et al., 2004), the large number of available samples now allows pursuing an approach with higher 

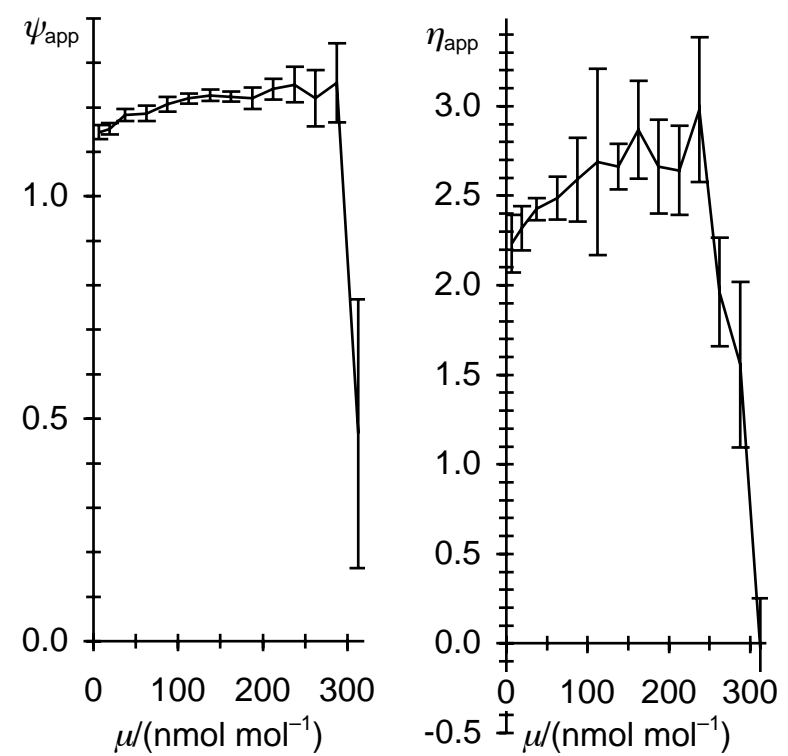

Fig. 13. Dependence of binned $\psi$ - and $\eta$-values on the $\mathrm{N}_{2} \mathrm{O}$ mixing ratio. Error bars correspond to the standard error of the mean for the respective mixing ratio bin.

resolution. We compute $\eta_{\text {app }}$ and $\psi_{\text {app }}$ values directly for each individual sample, using the isotope values normalized to a single tropopause date of 15 March 2002. Using $\eta_{\text {app }}$ and $\psi_{\text {app }}$ specifically in this combination of isotope signatures as chemical fingerprints ensures that quantities with comparable errors are combined, in order to minimize statistical errors. By plotting $\ln \left(1+{ }^{2} \delta^{15} \mathrm{~N}\right)$ vs. $\ln \left(1+{ }^{1} \delta^{15} \mathrm{~N}\right)$ and $\ln \left(1+\delta^{15} \mathrm{~N}\right)$ vs. $\ln \left(1+\delta^{18} \mathrm{O}\right)$, it was verified that no significant offsets from the origin remained for samples with low enrichment. The normalization procedure was therefore effective in removing any offsets, which might otherwise have affected the computed $\eta_{\text {app }}$ and $\psi_{\text {app }}$ values. The results were then binned in $25 \mathrm{nmol} / \mathrm{mol}$ intervals and are shown in Fig. 13.

Figure 4 illustrates the range of expected $\eta$ and $\psi$ values as a function of the contribution of photo-oxidation to the total $\mathrm{N}_{2} \mathrm{O}$ sink, computed using the following equation

$\eta=\frac{x^{2} \varepsilon^{15} \mathrm{~N}_{\text {oxid }}+(1-x)^{2} \varepsilon^{15} \mathrm{~N}_{\text {photo }}}{x{ }^{1} \varepsilon^{15} \mathrm{~N}_{\text {oxid }}+(1-x){ }^{1} \varepsilon^{15} \mathrm{~N}_{\text {photo }}}$

and similarly for $\psi$.

Since the influence of transport is effectively removed by using ratios of fractionation constants, rather than the fractionation constants themselves, the contribution of photooxidation can be derived either graphically from Fig. 14 or numerically by identifying $\eta$ with $\eta_{\text {app }}$ and solving Eq. (9) for $x$ :

$x=\left(1+\frac{{ }^{1} \varepsilon^{15} \mathrm{~N}_{\text {oxid }}}{{ }^{1}{ }^{15} \mathrm{~N}_{\text {photo }}} \frac{\eta_{\text {app }}-\eta_{\text {oxid }}}{\eta_{\text {photo }}-\eta_{\text {app }}}\right)^{-1}$

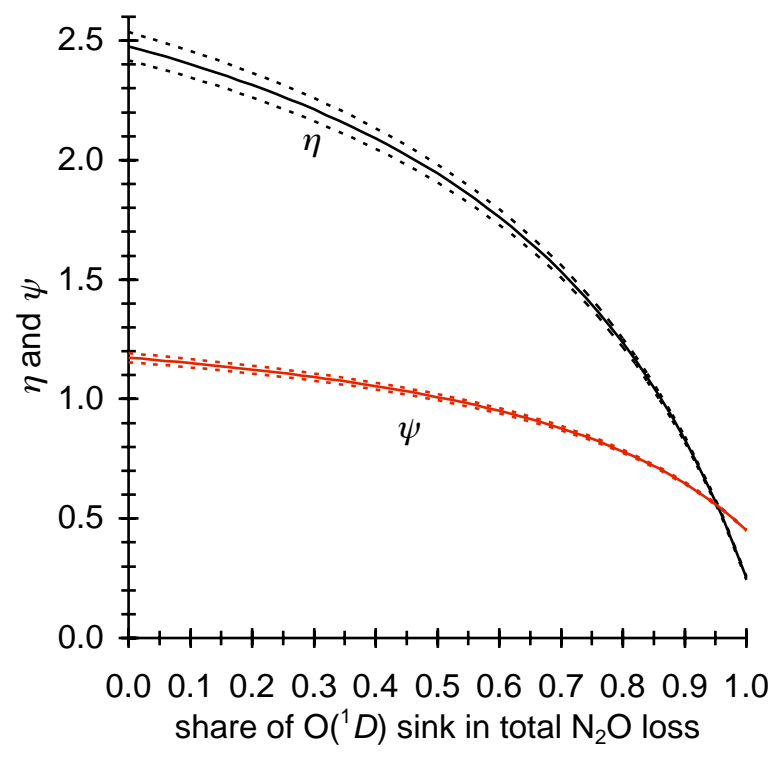

Fig. 14. Dependence of $\eta$ - and $\psi$-values on the share of the $\mathrm{O}\left({ }^{1} \mathrm{D}\right)$ sink in total $\mathrm{N}_{2} \mathrm{O}$ loss. $\eta$ - and $\psi$ have been computed from Eq. (9) using fractionation constants measured for broadband photolysis at $233 \mathrm{~K}$ (Kaiser et al., 2002b) and reaction of $\mathrm{N}_{2} \mathrm{O}$ with $\mathrm{O}\left({ }^{1} \mathrm{D}\right)$ (Kaiser et al., 2002a). Uncertainties are indicated by dashed lines. Isotope fractionation by the broadband photolysis lamp used by Kaiser et al. (2002b) is in good agreement with isotope effects integrated over the stratospheric actinic flux using cross section data from von Hessberg et al. (2004), see also Sect. 3.2.3.

The small variation of $\varepsilon^{15} \mathrm{~N}_{\text {oxid }}$ with altitude can be neglected (Kaiser et al., 2002b). At $\mathrm{N}_{2} \mathrm{O}$ mixing ratios between 300 and $325 \mathrm{nmol} \mathrm{mol}^{-1}$, both $\eta_{\text {app }}$ and $\psi_{\text {app }}$ show low values, which approach the values expected for photo-oxidation alone. The $\eta_{\text {app }}$ values for the $275-300 \mathrm{nmol} \mathrm{mol}^{-1}$ and 250 $275 \mathrm{nmol} \mathrm{mol}^{-1}$ bins also show some contribution by the $\mathrm{O}\left({ }^{1} \mathrm{D}\right)$ sink, i.e., about $70 \%$ and $50 \%$, respectively. However, this is not confirmed by the results for $\psi_{\text {app. }}$. For mixing ratios below $250 \mathrm{nmol} \mathrm{mol}^{-1}$, there are no indications of any signficant $\mathrm{O}\left({ }^{1} \mathrm{D}\right)$ contribution, in contrast to the calculations of Toyoda et al. (2004), which indicated a contribution of between 70 and $80 \%$ for mixing ratios down to about $175 \mathrm{nmol} \mathrm{mol}^{-1}$. In fact, $\eta_{\text {app }}$ and $\psi_{\text {app }}$ even exceed the values expected for photolysis slightly, possibly indicating a discrepancy to laboratory measurements. The $\eta_{\text {app }}$ values have larger analytical errors than the $\psi_{\text {app }}$ values, even though they should in principle be more sensitive to variations of the $\mathrm{O}\left({ }^{1} \mathrm{D}\right)$ contribution to the total $\mathrm{N}_{2} \mathrm{O}$ sink, because its end-member values span a larger range than for $\psi$. At least for $\mathrm{N}_{2} \mathrm{O}$ mixing ratios $>300 \mathrm{nmol} \mathrm{mol}^{-1}$, the results shown here indicate that a much larger fraction than $10 \%$ is removed by photo-oxidation (cf. Introduction). This was initially suggested by Röckmann et al. (2001).

At low $\mathrm{N}_{2} \mathrm{O}$ mixing ratios, both $\eta$ and $\psi$ decline. This is expected from the temperature dependence of $\eta$ and $\psi$ in 
$\mathrm{N}_{2} \mathrm{O}$ photolysis (Kaiser et al., 2002b) which leads to $\eta$ and $\psi$ decreases due to the gradual increase of stratospheric temperatures with altitude. Stratospheric spectral ultraviolet variations are too small to cause effects due to the wavelength dependence of $\mathrm{N}_{2} \mathrm{O}$ isotope fractionation (Kaiser et al., 2003b).

Note that the $\eta$ and $\psi$ values shown here do not correspond to their local values at the point where the sample was obtained, but are convoluted with the $\eta$ and $\psi$ variations at higher mixing ratios. Nevertheless, any decrease in the local $\eta$ and $\psi$ values will necessarily also show up in the "globally" calculated values shown here. We tried to derive $\eta$ and $\psi$ variations from polynomial fits to plots of $\ln \left(1+{ }^{2} \delta^{15} \mathrm{~N}\right)$ vs. $\ln \left(1+{ }^{1} \delta^{15} \mathrm{~N}\right)$ and $\ln \left(1+\delta^{15} \mathrm{~N}\right)$ vs. $\ln \left(1+\delta^{18} \mathrm{O}\right)$. However, this requires polynomial fits of at least third order, with associated large uncertainties in the regression coefficients. The polynomial fits show negative curvature, i.e., $\eta$ and $\psi$ decrease with increasing ${ }^{1} \delta^{15} \mathrm{~N}$ or $\delta^{18} \mathrm{O}$ values. However, the uncertainties of the calculated inflection point from this analysis are too high to allow for detecting a change in curvature near the origin.

In conclusion from the position-dependent $\mathrm{N}_{2} \mathrm{O}$ isotope measurements, it seems now that they do bear an unambiguous signature of varying contributions of photolysis and photo-oxidation as tentatively established previously (Kaiser, 2002; Röckmann et al., 2001). We also searched for meridional variations in $\eta$ or $\psi$ by binning the data into polar, midlatitude and tropical samples. However, no significant meridional contrasts were found and we attribute the suggested lower tropical $\eta$ values derived from Park et al.'s (2004) reanalysis of our previously published subset of the India 04/99 samples (Röckmann et al., 2001) to analytical artifacts.

\section{Conclusions}

Apparent fractionation constants $\left(\varepsilon_{\text {app }}\right)$ for stratospheric $\mathrm{N}_{2} \mathrm{O}$ are shown to be dependent on the time of sampling (season and year), latitude and altitude. $\left|\varepsilon_{\text {app }}\right|$ is highest near the equator and decreases towards the poles. Seasonal differences are difficult to discern at polar and tropical latitudes because of limited data coverage, but point to higher $\left|\varepsilon_{\text {app }}\right|$ values in fall than in late spring/summer. This can be attributed largely to transport effects (Morgan et al., 2004), with faster transport time-scales in the tropics and in fall leading to higher apparent fractionation constants of greater magnitude. Modeling attempts therefore have to consider seasonal and meridional variations of the $\mathrm{N}_{2} \mathrm{O}$ isotope signature if quantitative agreement with observations is to be achieved. It might even be necessary to include short-term temporal or longitudinal variations as a comparison between the Gap 06/99 and Sanriku 05/99 profiles shows (Fig. 8 and Sect. 3.2).

In the lower stratosphere (mixing ratios above $200 \mathrm{nmol} \mathrm{mol}^{-1}$ ), $\mathrm{N}_{2} \mathrm{O}$ isotope and mixing ratios display a compact relationship, which can be exploited for calculations of the atmospheric $\mathrm{N}_{2} \mathrm{O}$ isotope budget (Kaiser et al., 2004b; Park et al., 2004; Röckmann et al., 2003a). In spite of this compact relationship, lower stratospheric $\mathrm{N}_{2} \mathrm{O}$ clearly shows a fingerprint of photo-oxidation in its isotopic signature, as diagnosed from correlations between individual isotope signatures. Stronger variations in the correlation between $\delta^{15} \mathrm{~N}, \delta^{18} \mathrm{O}$ and the position-dependent $\delta^{15} \mathrm{~N}$ values can be unambiguously detected in the upper stratosphere. These might be explained by temperature effects (Kaiser, 2002; Kaiser et al., 2002b).

We have also discussed conceptual models to rationalize stratospheric $\mathrm{N}_{2} \mathrm{O}$ isotope variations in the framework of modified Rayleigh fractionation (considering a onedimensional reaction-diffusion-advection scheme), two-endmember mixing and continuous weak mixing between intravortex and extravortex air (Plumb et al., 2000). None of the models captures all stratospheric features. Especially, aircraft samples from the polar vortex at $\mathrm{N}_{2} \mathrm{O}$ mixing ratios below $200 \mathrm{nmol} \mathrm{mol}^{-1}$ deviate both from isotope variations expected for Rayleigh fractionation and two-end-member mixing, but could be explained by continuous weak mixing between extravortex and intravortex air. More detailed threedimensional model simulations will be needed to fully appraise the expanding set of stratospheric $\mathrm{N}_{2} \mathrm{O}$ isotope measurements. A table with station information, $\mathrm{N}_{2} \mathrm{O}$ mixing and isotope ratios is available on request from the authors.

Acknowledgements. We would like to thank B. Knape for help with the mass-spectrometric measurements and A. Plumb for useful discussions about a conceptual model of continuous weak mixing. Thanks to $\mathrm{M}$. Braß for providing $\mathrm{CH}_{4}$ mixing ratios and to D. Griffith for useful discussions on slopes in Rayleigh fractionation plots. Three anonymous reviewers are acknowledged for constructive comments, which helped improve the original discussion paper. The ISOSTRAT project in Heidelberg was funded by the BMBF within the AFO2000 project (grant 07ATC01). We thank CNES for excellent performance of the mid- and high-latitude balloon launches. Funding from various projects of ESA, DLR, BMBF and the European Commission is gratefully acknowledged for the balloon operations.

Edited by: U. Pöschl

\section{References}

Bender, M. L.: The $\delta^{18} \mathrm{O}$ of dissolved $\mathrm{O}_{2}$ in seawater: A unique tracer of circulation and respiration in the deep sea, J. Geophys. Res., 95, 22 243-22 252, 1990.

Bernard, S., Röckmann, T., Kaiser, J., Barnola, J.-M., Fischer, H., Blunier, T., and Chappellaz, J.: Constraints on $\mathrm{N}_{2} \mathrm{O}$ budget changes since pre-industrial time from new firn air and ice core isotope measurements, Atmos. Chem. Phys., 6, 493-503, 2006, http://www.atmos-chem-phys.net/6/493/2006/.

Boering, K. R., Wofsy, S. C., Daube, B. C., Schneider, H. R., Loewenstein, M., Podolske, J. R., and Conway, T. J.: Strato- 
spheric mean ages and transport rates from observations of carbon dioxide and nitrous oxide, Science, 274, 1340-1343, 1996.

Brenninkmeijer, C. A. M. and Röckmann, T.: Mass spectrometry of the intramolecular nitrogen isotope distribution of environmental nitrous oxide using fragment-ion analysis, Rapid Commun. Mass Spectrom., 13, 2028-2033, 1999.

Brenninkmeijer, C. A. M., Janssen, C., Kaiser, J., Röckmann, T., Rhee, T. S., and Assonov, S. S.: Isotope effects in the chemistry of atmospheric trace compounds, Chem. Rev., 103, 5125-5162, 2003.

Curtius, J., Weigel, R., Vssing, H.-J., Wernli, H., Werner, A., Volk, C.-M., Konopka, P., Krebsbach, M., Schiller, C., Roiger, A., Schlager, H., Dreiling, V., and Borrmann, S.: Observations of meteoric material and implications for aerosol nucleation in the winter Arctic lower stratosphere derived from in situ particle measurements, Atmos. Chem. Phys., 5, 3053-3069, 2006, http://www.atmos-chem-phys.net/5/3053/2006/.

Engel, A., Strunk, M., Müller, M., Haase, H.-P., Poss, C., Levin, I., and Schmidt, U.: Temporal development of total chlorine in the high-latitude stratosphere based on reference distributions of mean age derived from $\mathrm{CO}_{2}$ and $\mathrm{SF}_{6}$, J. Geophys. Res., 107, 1$11,2002$.

Engel, A., Möbius, T., Haase, H.-P., Bönisch, H., Wetter, T., Schmidt, U., Levin, I., Reddmann, T., Oelhaf, H., Grunow, K., Huret, N., and Pirre, M.: Observation of mesospheric air inside the arctic stratospheric polar vortex in early 2003, Atmos. Chem. Phys., 6, 267-282, 2006, http://www.atmos-chem-phys.net/6/267/2006/.

Esler, M. B., Griffith, D. W. T., Wilson, S. R., and Steele, L. P.: Precision trace gas analysis by FT-IR spectroscopy. 1. Simultaneous analysis of $\mathrm{CO}_{2}, \mathrm{CH}_{4}, \mathrm{~N}_{2} \mathrm{O}$, and $\mathrm{CO}$ in air, Anal. Chem., 72, 206-215, 2000.

Griffith, D. W. T., Toon, G. C., Sen, B., Blavier, J.-F., and Toth, R. A.: Vertical profiles of nitrous oxide isotopomer fractionation measured in the stratosphere, Geophys. Res. Lett., 27, 24852488, 2000.

Johnston, J. C., Cliff, S. S., and Thiemens, M. H.: Measurement of multioxygen isotopic $\left(\delta^{18} \mathrm{O}\right.$ and $\left.\delta^{17} \mathrm{O}\right)$ fractionation factors in the stratospheric sink reactions of nitrous oxide, J. Geophys. Res., 100, 16 801-16804, 1995.

Kaiser, J.: Stable isotope investigations of atmospheric nitrous oxide (http://archimed.uni-mainz.de/pub/2003/0004/), Ph.D. thesis, Johannes Gutenberg-Universität, Mainz, 2002.

Kaiser, J., Brenninkmeijer, C. A. M., and Röckmann, T.: Intramolecular ${ }^{15} \mathrm{~N}$ and ${ }^{18} \mathrm{O}$ fractionation in the reaction of $\mathrm{N}_{2} \mathrm{O}$ with $\mathrm{O}\left({ }^{1} \mathrm{D}\right)$ and its implications for the stratospheric $\mathrm{N}_{2} \mathrm{O}$ isotope signature, J. Geophys. Res., 107, 4214, doi:10.1029/2001JD001506, 2002a.

Kaiser, J., Röckmann, T., and Brenninkmeijer, C. A. M.: Temperature dependence of isotope fractionation in $\mathrm{N}_{2} \mathrm{O}$ photolysis, Phys. Chem. Chem. Phys., 4, 4220-4230, doi:10.1039/B204837J, 2002b.

Kaiser, J., Röckmann, T., and Brenninkmeijer, C. A. M.: Complete and accurate mass-spectrometric isotope analysis of tropospheric nitrous oxide, J. Geophys. Res., 108, 4476, doi:10.1029/2003JD003613, 2003a.

Kaiser, J., Röckmann, T., Brenninkmeijer, C. A. M., and Crutzen, P. J.: Wavelength dependence of isotope fractionation in $\mathrm{N}_{2} \mathrm{O}$ photolysis, Atmos. Chem. Phys., 3, 303-313, 2003 b.
Kaiser, J., Park, S., Boering, K. A., Brenninkmeijer, C. A. M., Hilkert, A. W., and Röckmann, T.: Mass-spectrometric method for the absolute calibration of the intramolecular nitrogen isotope distribution in nitrous oxide, Anal. Bioanal. Chem., 378, 256269, doi:10.1007/s00216-003-2233-2, 2004a.

Kaiser, J., Röckmann, T., and Brenninkmeijer, C. A. M.: Contribution of mass-dependent fractionation to the oxygen isotope anomaly of atmospheric nitrous oxide, J. Geophys. Res., 109, D03305, doi:10.1029/2003JD004088, 2004b.

Kaiser, J. and Röckmann, T.: Absence of isotope exchange in the reaction of $\mathrm{N}_{2} \mathrm{O}+\mathrm{O}\left({ }^{1} \mathrm{D}\right)$ and the global $\Delta^{17} \mathrm{O}$ budget of nitrous oxide, Geophys. Res. Lett., 32, L15808, doi:10.1029/2005GL023199, 2005.

Kaye, J. A.: Mechanisms and observations for isotope fractionation of molecular species in planetary atmospheres, Rev. Geophys., 25, 1609-1658, 1987.

Keeling, C. D.: The concentration and isotopic abundance of atmospheric carbon dioxide in rural areas, Geochim. Cosmochim. Acta, 13, 322-334, 1958.

Kim, K.-R. and Craig, H.: Nitrogen-15 and oxygen-18 characteristics of nitrous oxide: A global perspective, Science, 262, 18551857, 1993.

McLinden, C. A., Prather, M. J., and Johnson, M. S.: Global modeling of the isotopic analogues of $\mathrm{N}_{2} \mathrm{O}$ : Stratospheric distributions, budgets, and the ${ }^{17} \mathrm{O}-{ }^{18} \mathrm{O}$ mass-independent anomaly, J. Geophys. Res., 108, 4233, doi:10.1029/2002JD002560, 2003.

Michelsen, H. A., Manney, G. L., Gunson, M. R., Rinsland, C. P., and Zander, R.: Correlations of stratospheric abundances of $\mathrm{CH}_{4}$ and $\mathrm{N}_{2} \mathrm{O}$ derived from ATMOS measurements, Geophys. Res. Lett., 25, 2777-2780, 1998.

Minschwaner, K., Salawitch, R. J., and McElroy, M. B.: Absorption of solar radiation by $\mathrm{O}_{2}$ : Implications for $\mathrm{O}_{3}$ and lifetimes of $\mathrm{N}_{2} \mathrm{O}, \mathrm{CFCl}_{3}$, and $\mathrm{CF}_{2} \mathrm{Cl}_{2}$, J. Geophys. Res., 98, 10 543-10 561, 1993.

Moore, H.: Isotopic measurement of atmospheric nitrogen compounds, Tellus, 26, 169-174, 1974.

Morgan, C. G., Allen, M., Liang, M. C., Shia, R. L., Blake, G. A., and Yung, Y. L.: Isotopic fractionation of nitrous oxide in the stratosphere: Comparison between model and observations, J. Geophys. Res., 109, D04305, doi:10.1029/2003JD003402, 2004.

Park, S., Atlas, E. L., and Boering, K. A.: Measurements of $\mathrm{N}_{2} \mathrm{O}$ isotopologues in the stratosphere: Influence of transport on the apparent enrichment factors and the isotope fluxes to the troposphere, J. Geophys. Res., 109, D01305, doi:10.1029/2003JD003731, 2004.

Patra, P. K., Lal, S., Venkataramani, S., and Chand, D.: Halogen Occultation Experiment (HALOE) and balloon-borne in situ measurements of methane in stratosphere and their relation to the quasi-biennial oscillation (QBO), Atmos. Chem. Phys., 3, 10511062, 2003, http://www.atmos-chem-phys.net/3/1051/2003/.

Plumb, R. A., Waugh, D. W., and Chipperfield, M. P.: The effects of mixing on tracer relationships in the polar vortices, J. Geophys. Res., 105, 10 047-10 062, 2000.

Prather, M., Ehhalt, D., Dentener, F., Derwent, R., Dlugokencky, E., Holland, E., Isaksen, I., Katima, J., Kirchhoff, V., Matson, P., Midgley, P., and Wang, M.: Atmospheric chemistry and greenhouse gases, in: Climate Change 2001: The Scientific Basis, edited by: Houghton, J. T., Ding, Y., Griggs, D. J., et al., 239- 
287, Cambridge University Press, 2001.

Prinn, R. G., Weiss, R. F., Fraser, P. J., Simmonds, P. G., Cunnold, D. M., Alyea, F. N., O'Doherty, S., Salameh, P., Miller, B. R., Huang, J., Wang, R. H. J., Hartley, D. E., Harth, C., Steele, L. P., Sturrock, G., Midgley, P. M., and McCulloch, A.: A history of chemically and radiatively important gases in air deduced from ALE/GAGE/AGAGE, J. Geophys. Res., 105, 17 751-17 792, 2000.

Rahn, T. and Wahlen, M.: Stable isotope enrichment in stratospheric nitrous oxide, Science, 278, 1776-1778, 1997.

Rahn, T., Zhang, H., Wahlen, M., and Blake, G. A.: Stable isotope fractionation during ultraviolet photolysis of $\mathrm{N}_{2} \mathrm{O}$, Geophys. Res. Lett., 25, 4489-4492, 1998.

Röckmann, T., Kaiser, J., Brenninkmeijer, C. A. M., Crowley, J. N., Borchers, R., Brand, W. A., and Crutzen, P. J.: The isotopic enrichment of nitrous oxide $\left({ }^{15} \mathrm{~N}^{14} \mathrm{NO},{ }^{14} \mathrm{~N}^{15} \mathrm{NO},{ }^{14} \mathrm{~N}^{14} \mathrm{~N}^{18} \mathrm{O}\right)$ in the stratosphere and in the laboratory, J. Geophys. Res., 106, 10 403-10 410, 2001.

Röckmann, T., Kaiser, J., and Brenninkmeijer, C. A. M.: The isotopic fingerprint of the pre-industrial and the anthropogenic $\mathrm{N}_{2} \mathrm{O}$ source, Atmos. Chem. Phys., 3, 315-323, 2003 a.

Röckmann, T., Kaiser, J., Brenninkmeijer, C. A. M., and Brand, W. A.: Gas-chromatography/isotope-ratio mass spectrometry method for high-precision position-dependent ${ }^{15} \mathrm{~N}$ and ${ }^{18} \mathrm{O}$ measurements of atmospheric nitrous oxide, Rapid Commun. Mass Spectrom., 17, 1897-1908, 2003b.

Röckmann, T. and Levin, I.: High-precision determination of the changing isotopic composition of atmospheric $\mathrm{N}_{2} \mathrm{O}$ from 1990 to 2002, J. Geophys. Res., 110, D21304, doi:10.1029/2005JD006066, 2005.

Schmidt, U., Kulessa, G., Klein, E., Roth, E. P., Fabian, P., and Borchers, R.: Intercomparison of balloon-borne cryogenic whole air samplers during the MAP/GLOBUS 1983 campaign, Planet. Space Sci., 35, 647, 1987.

Selwyn, G. S. and Johnston, H. S.: Ultraviolet absorption spectrum of nitrous oxide as function of temperature and isotopic substitution, J. Chem. Phys., 74, 3791-3803, 1981.
Sowers, T., Rodebaugh, A., Yoshida, N., and Toyoda, S.: Extending records of the isotopic composition of atmospheric $\mathrm{N}_{2} \mathrm{O}$ back to 1800 A.D. from air trapped in snow at the South Pole and the Greenland Ice Sheet Project II ice core, Global Biogeochem. Cycles, 16, 1129, doi:10.1029/2001GB001911, 2002.

Toyoda, S. and Yoshida, N.: Determination of nitrogen isotopomers of nitrous oxide on a modified isotope ratio mass spectrometer, Anal. Chem., 71, 4711-4718, 1999.

Toyoda, S., Yoshida, N., Urabe, T., Aoki, S., Nakazawa, T., Sugawara, S., and Honda, H.: Fractionation of $\mathrm{N}_{2} \mathrm{O}$ isotopomers in the stratosphere, J. Geophys. Res., 106, 7515-7522, 2001.

Toyoda, S., Yoshida, N., Urabe, T., Nakayama, Y., Suzuki, T., Tsuji, K., Shibuya, K., Aoki, S., Nakazawa, T., Ishidoya, S., Ishijima, K., Sugawara, S., Machida, T., Hashida, G., Morimoto, S., and Honda, H.: Temporal and latitudinal distributions of stratospheric $\mathrm{N}_{2} \mathrm{O}$ isotopomers, J. Geophys. Res., 109, D08308, doi:10.1029/2003JD004316, 2004.

Volk, C. M., Elkins, J. W., Fahey, D. W., Dutton, G. S., Gilligan, J. M., Loewenstein, M., Podolske, J. R., Chan, K. R., and Gunson, M. R.: Evaluation of source gas lifetimes from stratospheric observations, J. Geophys. Res., 102, 25 543-25 563, 1997.

von Hessberg, P., Kaiser, J., Enghoff, M. B., McLinden, C. A., Sorensen, S. S., Röckmann, T., and Johnson, M. S.: Ultra-violet absorption cross sections of isotopically substituted nitrous oxide species: ${ }^{14} \mathrm{~N}^{14} \mathrm{NO},{ }^{15} \mathrm{~N}^{14} \mathrm{NO},{ }^{14} \mathrm{~N}^{15} \mathrm{NO}$ and ${ }^{15} \mathrm{~N}^{15} \mathrm{NO}$, Atmos. Chem. Phys., 4, 1237-1253, 2004, http://www.atmos-chem-phys.net/4/1237/2004/.

Yoshida, N., Morimoto, H., and Matsuo, S.: UV photolysis and microbial reduction as major sinks of nitrous oxide with emphasis on kinetic nitrogen isotope discrimination, Eos Trans. AGU, 71, 933-934, 1990.

Yoshida, N. and Toyoda, S.: Constraining the atmospheric $\mathrm{N}_{2} \mathrm{O}$ budget from intramolecular site preference in $\mathrm{N}_{2} \mathrm{O}$ isotopomers, Nature, 405, 330-334, 2000.

Yung, Y. L. and Miller, C. E.: Isotopic fractionation of stratospheric nitrous oxide, Science, 278, 1778-1780, 1997. 